\title{
Theoretical and Experimental Investigation of Electron Transfer Mediated Decay in ArKr Clusters
}

\author{
Elke Fasshauer ${ }^{\mathrm{a}}$, Marko Förstel ${ }^{\mathrm{b}, 1}$, Melanie Mucke ${ }^{\mathrm{b}, 2}$, Tiberiu Arion ${ }^{\mathrm{b}, 3}$, Uwe \\ Hergenhahn ${ }^{\mathrm{c}, \mathrm{d}}$ \\ ${ }^{a}$ Centre for Theoretical and Computational Chemistry, Department of Chemistry, \\ University of Tromsø- The Arctic University of Norway, N-9037 Tromsø, Norway \\ ${ }^{b}$ Max-Planck-Institute for Plasma Physics, Boltzmannstr. 2, 85748 Garching, Germany \\ ${ }^{c}$ Leibniz Institute of Surface Modification, Permoserstr. 15, 04318 Leipzig, Germany \\ ${ }^{d}$ Max-Planck-Institute for Plasma Physics, Wendelsteinstr. 1, 14791 Greifswald, \\ Germany
}

\section{Abstract}

We investigate the electronic decay of an $\mathrm{Ar} 3 \mathrm{~s}^{-1}$ vacancy in medium sized ArKr clusters. The only energetically accessible, radiationless decay mechanism is Electron Transfer Mediated Decay Three (ETMD3). Here, the argon vacancy is filled by an electron from one krypton atom, and the excess energy is transferred to a second krypton atom which consequently emits an electron. For the theoretical calculation of ETMD3 spectra, in a bottom-up approach, we study the dependence of the decay width on the geometry of elementary sets of three atoms, from which any cluster can be composed. We simulate the ETMD3 spectra of medium sized ArKr clusters and compare

Email addresses: elke.fasshauer@uit.no (Elke Fasshauer), uwe.hergenhahn@iom-leipzig. de (Uwe Hergenhahn)

${ }^{1}$ Now at: Institut für Optik und Atomare Physik, Technische Universität Berlin, Hardenbergstr. 36, 10623 Berlin, Germany

${ }^{2}$ Now at: Department of Physics and Astronomy, Uppsala University, Box 516, 75120 Uppsala, Sweden

${ }^{3}$ Now at: Center for Free-Electron Laser Science/DESY, Notkestr. 85, 22607 Hamburg, Germany 
the resulting spectra to experimental ETMD electron spectra presented earlier (Phys. Rev. Lett. 106, 033402 (2011)) and in this work. We show that ETMD3 is the dominating relaxation mechanism for the cases studied here. Keywords:

Electron Transfer Mediated Decay, ETMD3, Heterogeneous Noble Gas Clusters, Electronic Decay Process, ArKr

1 1. Introduction

2 A multi-electron system containing a vacancy in a level other than its outer valence shell is in an excited state, and will relax via emission of a pho4 ton, coupling to the nuclear motion or via an electronic decay processes. In 5 the latter case, the vacancy is filled by a valence electron and the excess en6 ergy is used to emit another electron. Depending on how the initial vacancy, 7 the electron that was filling it, and the emitted electron are distributed over

8 the atoms or molecules involved in the process, the process will have different 9 characteristics, like energetics and lifetimes.

If the initially ionized orbital $X_{i n}$, the electron donating orbital $X_{D}$ and the electron emitting orbital $X_{E}$ are all on one and the same atom or molecule, the process is called Auger decay, first observed by Meitner [1] and named after Auger [2]. Auger decay is element specific and therefore used for surface analysis in metallurgy, quality analysis of microelectronics $[3,4]$ and studies of heterogeneous catalysis [5]. In order to initiate this process, typically $X_{i n}$ has to have a vacancy in a core level. This requires a high ionization or excitation energy to create the initial vacancy, which limits the applicability of Auger decay to systems which do not suffer from radiation 
damage and therefore excludes most organic compounds. The Auger process has lifetimes in the as to fs range and, if energetically accessible, is in most cases the dominant relaxation mechanism.

Since 1997, decay processes in which the units are spread over different atoms or molecules, have been investigated [6-11]. The most basic of these is the so-called Interatomic / Intermolecular Coulombic Decay (ICD), in which the orbitals $X_{i n}$ and $X_{D}$ reside on one atom or molecule (subsystem $S_{1}$ ), while the excess energy is transferred to a different atom or molecule (subsystem $S_{2}$ ) which then emits an electron into the continuum: the ICD electron. In the final state of the process, the two positively charged subsystems repell each other and undergo Coulomb explosion. Meanwhile, different variants of ICD are known, in which the vacancy is created by excitation instead of ionization (resonant ICD) [12-16] or by Auger decay followed by ICD [1721]. In both cases the subsystems are characterized by a different number and distribution of charges after the decay, compared to the classical ICD. The ICD process is investigated in quantum dots for its use in the development of a new generation of IR detectors [22], catalyzing mechanisms of proteins [23], as a cause of DNA damage in radioation therapy [24] and as a possibility to destroy malignant tissue $[25,26]$. Its lifetime is normally in the order of femtoseconds, but strongly depends on the character of the decay partners involved, and on the environment of the originally ionized or excited site, since the decay width $(\Gamma=\hbar / \tau)$ scales at least linearly with the number of equidistant neighbours [27-30]. It has also been shown that not only the direct neighbours, but also atoms or molecules at larger distances may play a role in the overall process and may further increase the decay width [29, 31]. 
Yet other types of radiationless decay processes result, if the orbital ionized initially, and the one donating the electron that fills the vacancy, reside on different atoms or molecules. These processes are called Electron Transfer Mediated Decay (ETMD); and, since they involve an electron transfer step, normally proceed slower than Auger decay or ICD. They are therefore preferentially observed if the other decay processes are not accessible, or if many decay partners are available, which boosts the decay width of the ETMD. Depending on the character of the final state and the number of atoms or molecules involved, the ETMD is called ETMD3 (all three units spread over different atoms or molecules) [32], ETMD2 ( $X_{D}$ and $X_{E}$ coincide) [33] or exchange ICD ( $X_{i n}$ and $X_{E}$ coincide) [9,34]. The ETMD3 process is sketched in Fig. 1. The enhancement of double ionization cross sections near threshold due to the environment was explained by ETMD2 starting with the single ionization of one of the surrounding atoms/molecules [35]. Recently, the radiation damage of metal complexes after exposure to x-rays was assigned to the result from several different ETMD mechanisms [36].

All three variants of electron transfer mediated decay processes were found in experiments (ETMD3 e.g. in [37, 38], ETMD2 in [39], exchange ICD in $[9,20])$. In this work, we will focus on the first experimental demonstration of ETMD3, which was shown to be an important relaxation mechanism of Ar $3 \mathrm{~s}^{-1}$ inner valence vacancies in ArKr clusters [37]. Following the short presentation in [37], in this paper we will present a comprehensive account of our experimental results for this system, and will present extensive simulations of the decay process.

Already before the experiment, it was predicted theoretically that the 
ETMD3 channel is open after Ar 3s ionization in $\mathrm{Kr}-\mathrm{Ar}-\mathrm{Kr}$ trimers [40]. Interestingly, these investigations of trimers showed that the channel opening for the process is geometry dependent. In case of the linear $\mathrm{Ar}-\mathrm{Kr}-\mathrm{Kr}$ and the bent structure the decay channel is closed, while for the linear $\mathrm{Kr}-\mathrm{Ar}$ Kr structure the energy of the doubly ionized final state is lower than the ionization energy of the initially ionized argon atom and hence, the channel opens. However, the decay process has never been investigated theoretically for a system of more than three atoms. Meanwhile, we developed a method to simulate ICD and ETMD3 spectra for large clusters based on the decomposition of the cluster into pairs and triples of atoms, with the full spectrum constructed from the summed-up contributions (decay energies and decay partial widths) from each of these sub-units [28]. This approach was tested successfully against experiments in NeAr and ArXe clusters, where in both of these cases the arguably most interesting result consisted in the occurence of ICD between the initial vacancy and atoms in its second coordination shell $[29,31,41]$. Here, we will therefore apply it to $\mathrm{ArKr}_{2}$ trimers, to understand the basics of the process, and to larger ArKr clusters, thereby showing the suitability of the method for the ETMD3 process.

ArKr clusters have been the subject of the investigation of ICD before: ICD was found after Auger decay of Ar 2p or Kr 3d vacancies [42, 43], and ICD induced after resonant Auger decay was discussed in Ref.s [44, 45]. These processes need much higher excitation energies than the experiment in Ref. [37] and in this paper, to which we will compare our theoretical results.

The outline of the paper is as follows: For completeness, we firstly repeat the essentials of our theoretical approach to the calculation of ETMD spec- 
94

tra, then we summarize some details of the experiment and discuss properties of the clusters that are produced. We then summarize some computational details specific to this project. In the main theoretical part, we delineate how the ETMD decay widths and energies depend on the geometrical arrangement of the three atoms involved in the decay, and on the orientation of their orbitals. After that, we describe the consequences for the spectra of larger ArKr clusters with either a strict Kr core-Ar shell arrangement, or an arrangement that allows for some diffusion of the Ar shell into the core. Finally we present experimental results on the ArKr systems in substantially more detail than in our earlier work [37], and make a comparison to the calculations. 

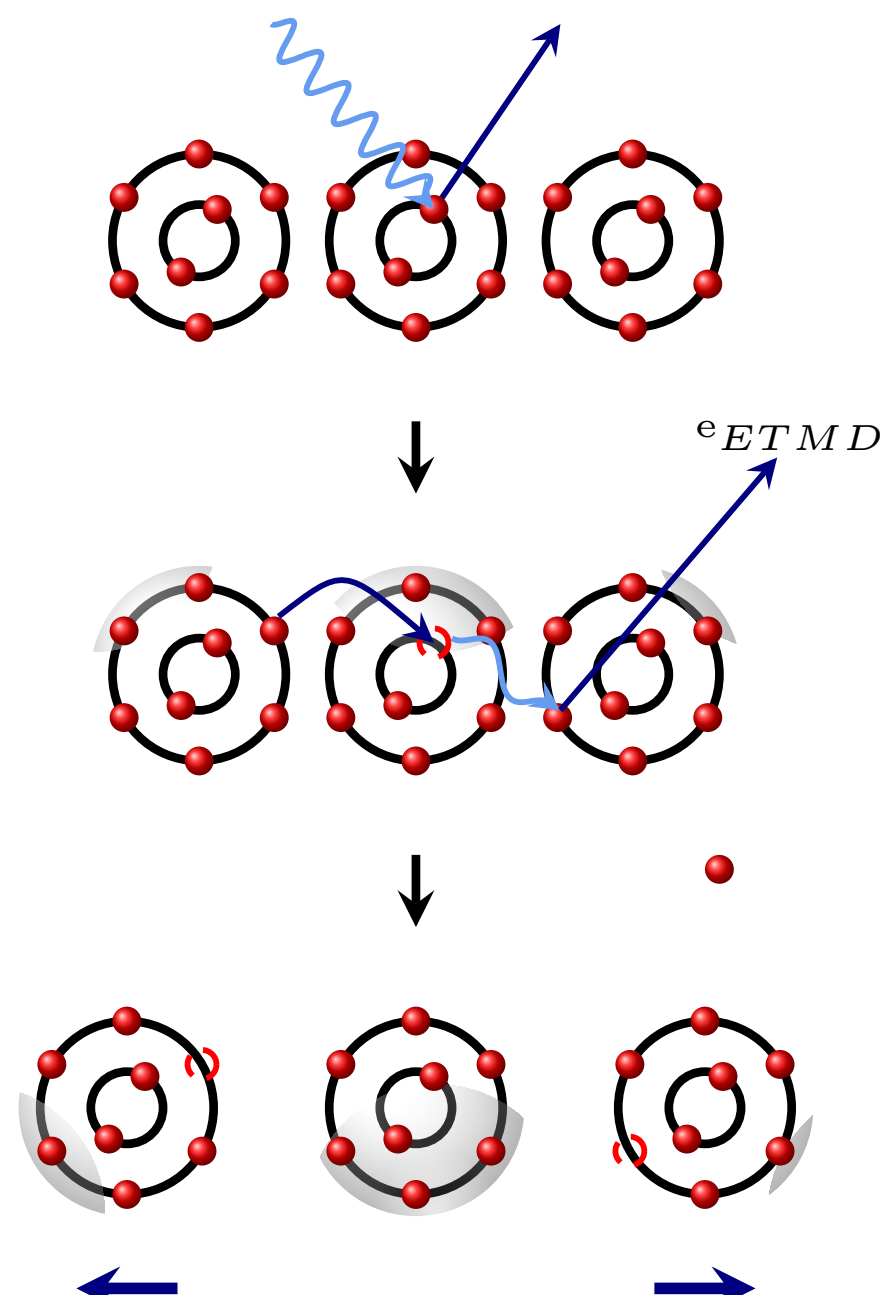

Figure 1: Sketch of an Electron Transfer Mediated Decay involving three atoms (ETMD3), initiated by photoionization. 


\section{Theory}

Whether an electronic decay process is observed, depends on its energetic accessibility and its efficiency and hence, its ability to compete with alternative decay mechanisms. We will discuss these two aspects separately.

In an electronic decay process, the energy of the initially ionized subsystem must be equal to the sum of final state's energy and the kinetic energy of the emitted electron, $E_{\mathrm{in}}=E_{\mathrm{fin}}+E_{\mathrm{sec}}$. Therefore, a process is only accessible energetically, if the energy of the final state $E_{\text {fin }}$ is lower than the energy of the initial state $E_{\text {in }}$. These two energy values can be approximated by the single ionization potentials $(S I P)$ of the different units, and, for processes in which the two finally ionized units $X_{D}$ and $X_{E}$ do not coincide on the same atom or molecule, the distance $d$ between $X_{D}$ and $X_{E}$ :

$$
\begin{aligned}
& E_{\mathrm{in}}=\operatorname{SIP}\left(X_{\mathrm{in}}\right) \\
& E_{\mathrm{fin}}^{\beta}=\operatorname{SIP}\left(X_{D}^{\beta}\right)+\operatorname{SIP}\left(X_{E}^{\beta}\right)+\frac{1}{d} \\
& E_{\mathrm{sec}}^{\beta}=E_{\mathrm{in}}^{\beta}-E_{\mathrm{fin}}^{\beta},
\end{aligned}
$$

where the index $\beta$ distinguished between different quantum states located on the same sub-unit. Atomic units are used.

For the case of ETMD3 in ArKr clusters, $d$ is the interatomic distance between the two ionized krypton atoms. These quantities depend on the decay channel under investigation. In the relativistic approach, which is necessary for the treatment of krypton due to spin-orbit coupling effects, these are characterized by the different total angular momenta $J_{D}$ and $J_{E}$ and their combinations. Since the allowed values for the angular momenta are $3 / 2$ and $1 / 2$, there are four conceivable decay channels $\beta$ : ArKr $4 \mathrm{p}_{3 / 2}^{-1} \mathrm{Kr} 4 \mathrm{p}_{3 / 2}^{-1}$, 
$\operatorname{ArKr} 4 \mathrm{p}_{3 / 2}^{-1} \mathrm{Kr} 4 \mathrm{p}_{1 / 2}^{-1}$, ArKr $4 \mathrm{p}_{1 / 2}^{-1} \mathrm{Kr} 4 \mathrm{p}_{3 / 2}^{-1}$ and $\operatorname{ArKr} 4 \mathrm{p}_{1 / 2}^{-1} \mathrm{Kr} 4 \mathrm{p}_{1 / 2}^{-1}$. The working equation for determining the energy of the secondary electron reads:

$$
E_{\mathrm{sec}}^{\beta}=S I P\left(X_{\mathrm{in}}\right)-S I P\left(X_{D}^{\beta}\right)-S I P\left(X_{E}^{\beta}\right)-\frac{1}{d} .
$$

A channel $\beta$ is closed if the kinetic energy of the emitted electron is smaller than zero $\left(E_{\mathrm{sec}}<0\right)$.

A measure for the efficiency of the decay is the decay width $\Gamma=\hbar / \tau$, which is inversely proportional to the lifetime $\tau$. Following Wentzel [46], Feshbach [47, 48] and Fano [49], it can be written as:

$$
\Gamma=\sum_{\beta} \Gamma_{\beta}=\sum_{\beta} 2 \pi\left|\left\langle\Phi|\hat{V}| \chi_{\beta, \varepsilon}\right\rangle\right|^{2} .
$$

Here, $|\Phi\rangle$ and $\left|\chi_{\beta, \varepsilon}\right\rangle$ denote the wavefunctions of the initial and final state, respectively, and $\hat{V}$ is the interaction operator. Formulating an asymptotic approximation for the ETMD3 within this ansatz leads to

$$
\begin{aligned}
\Gamma_{\beta}= & \frac{1}{R^{6}} \sum_{M_{i n, D^{\prime}}} 2\left[\left|\left\langle\tilde{D}_{x}\left(M_{i n, D^{\prime}}\right)\right\rangle\right|^{2}\left(2+\sin ^{2} \alpha\right)+\left|\left\langle\tilde{D}_{z}\left(M_{i n, D^{\prime}}\right)\right\rangle\right|^{2}\left(1+\cos ^{2} \alpha\right)\right] \\
& \cdot \frac{c \sigma^{\left(X_{E}\right)}\left(\omega_{v p}\right)}{2 \pi \omega_{v p}}
\end{aligned}
$$

where $\left\langle\tilde{D}_{x / z}\left(M_{i n, D^{\prime}}\right)\right\rangle$ denotes the transition dipole moment between the initially ionized unit (termed $A$ in our earlier papers) and the electron donor (formerly termed $B$ ) in either $\tilde{x}$ or $\tilde{z}$ direction, and $\omega_{v p}$ denotes the energy transferred to the electron emitting unit, often referred to as the transfer of a virtual photon $(v p)[28] . \sigma^{\left(X_{E}\right)}$ denotes the photoionization cross section of sub-unit $E$ at the photon energy $\omega_{v p}$.

Clearly, $\Gamma_{\beta}$ within this approximation depends on the spatial coordinates of the three involved sub-units (here, the initially ionized $\mathrm{Ar}$ and two $\mathrm{Kr}$ 
a)

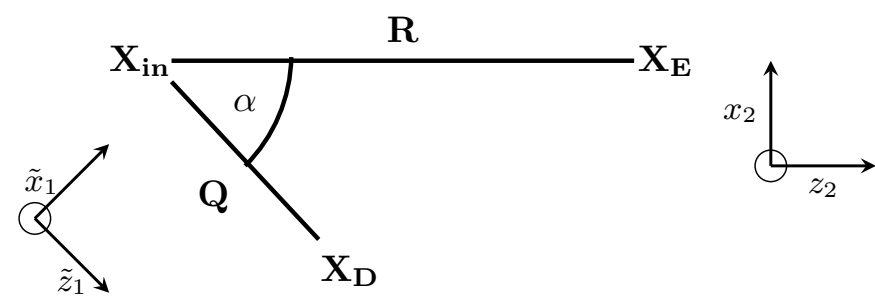

b)

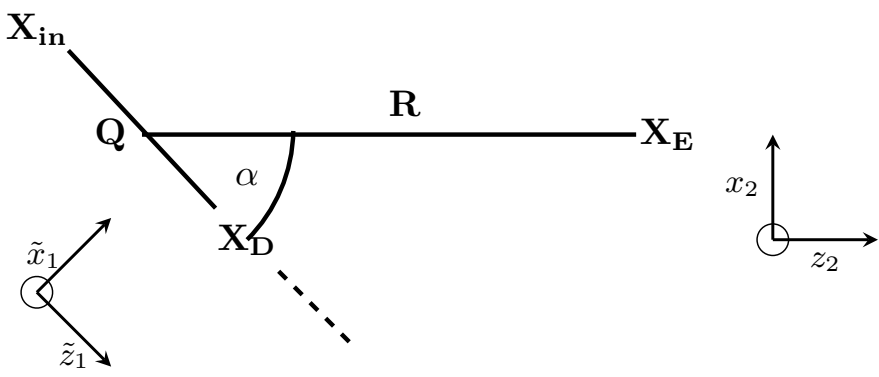

Figure 2: Different choices of Jacobi coordinates for the description of the geometry of the three centers involved in ETMD3. In panel a) the reference point for the energy transfer distance $R$ is chosen at the initially ionized unit $X_{i n}$, while in panel b) the reference point is chosen between $X_{i n}$ and $X_{D}$.

atoms). Implicit in Eq. (6), we describe these by the Jacobi coodinates of two distances and one angle, as shown in Fig. 2.

The two distances $Q$ and $R$ in Fig. 2 are inequivalent, as $Q$ is the distance of charge transfer between $X_{\text {in }}$ and $X_{\mathrm{D}}$, and $R$ is the distance over which the energy transfer, resulting in the ionization of $X_{\mathrm{E}}$, proceeds. Within our ansatz, it is of some consequence that the Jacobi coordinates can be chosen in different ways, as shown in the Figure. The endpoint for the energy 
transfer distance $R$ can be chosen anywhere on the connecting line between the initially ionized unit and the electron donor. Accordingly, for a given triple structure, also the value of the angle $\alpha$ changes. Which choice describes the process most accurately currently is not known. We note however that this choice affects the calculated results. In this paper, we choose the initially ionized unit as the anchor for the energy transfer distance $R$, corresponding to Fig. 2a, in order to avoid singularities, which can occur in cluster structures when $X_{E}$ is placed in-between $X_{i n}$ and $X_{D}$.

\section{Experimental}

The experiments were performed at the synchrotron radiation source BESSY II of Helmholtz-Zentrum Berlin, at the bending magnet beamline TGM4 and the undulator beamline UE112-PGM1. Our set-up is comprised of a vacuum chamber housing a cluster source in an expansion region and a magnetic bottle electron time-of-flight spectrometer in the detection region. These two regions are separated by a conical skimmer (Beam Dynamics) in order to maintain a pressure of approx. $5 \times 10^{-6}$ mbar in the detection region. The cluster source was described earlier in [50]. The magnetic bottle spectrometer and the coincident electron detection technique have also been described in detail $[50,51]$. In short, we produced homogeneous argon and krypton clusters as well as mixed ArKr clusters of different sizes and compositions by varying the expansion conditions and the initial argon - krypton mixing ratio. Gases were obtained commercially and used without further purification. The distance of the nozzle to the skimmer was varied to obtain the highest degree of condensation in each experiment and was in the order of 
a few mm. The skimmed cluster jet then passed the interaction region where it crossed a beam of monochromatic, horizontally polarized synchrotron radiation. Our magnetic bottle spectrometer was mounted perpendicular to the plane spanned by the light beam and the cluster jet, with the interaction region between the repelling magnet and the entrance aperture. Measurements of pure Ar clusters and ArKr clusters with 5\% admixture were done at the TGM4, remaining experiments at the UE112. In the experiments at TGM4, a conical nozzle with a diameter of $d=100 \mu m$ and a cone length of $t=1400 \mu \mathrm{m}$ was used. For the UE112 experiment the parameters were $d=80 \mu \mathrm{m}$ and $t=1100 \mu \mathrm{m}$. The half opening angle of the cone in both cases amounted to $\alpha=15^{\circ}$. Other experimental parameters are collected in Tab. 1.

The cluster size of homogeneous cluster can be estimated using an empirical scaling law [52]. This scaling law requires specific material constants $K_{c h}$, which are $K_{c h}(\mathrm{Ar})=1646$ for argon and $K_{c h}(\mathrm{Kr})=2980$ for krypton. In order to estimate the size of the inhomogeneous clusters we use a new material constant of the expanding mixture $K_{c h}(\mathrm{ArKr})$ which we derive using the partial krypton content, $p_{K r}$ of the mixture as follows: $K_{c h}(\mathrm{ArKr})=K_{c h}(\mathrm{Ar})\left(1-p_{K r}\right)+K_{c h}(\mathrm{Kr}) p_{K r}$. The resulting, estimated cluster size of the mixed clusters is given in the last column of Tab. 1, and is not very different from the size calculated for a pure Ar expansion (see also [53]).

The outer valence spectra of the mixed clusters serve us as a measure for the obtained cluster composition and are shown in Fig. 3. The outer valence spectra were taken at an ionization energy of $16.2 \mathrm{eV}$. Time-to-energy 
Table 1: The expansion parameters used for cluster production. Here, $\mathrm{Kr}_{\mathrm{in}}$ is the molar fraction of $\mathrm{Kr}$ in the expanding gas mixture, $T$ is the nozzle temperature, and $p$ the stagnation pressure. $\left\langle N_{\mathrm{Ar}}\right\rangle$ and $\left\langle N_{\mathrm{Kr}}\right\rangle$ refer to cluster sizes for a pure $\mathrm{Ar}$, or pure $\mathrm{Kr}$ expansion, respectively, calculated from the given expansion conditions via an empirical scaling law [52]. $\left\langle N_{\mathrm{ArKr}}\right\rangle$ uses an interpolation of the material specific parameters to arrive at a scaling law with some predictive power for mixed expansions, see text for details.

\begin{tabular}{lccccccc}
\hline & $\mathrm{Kr}_{\mathrm{in}}(\%)$ & $\mathrm{Kr}_{\mathrm{cl}}(\%)$ & $T(\mathrm{~K})$ & $p(\mathrm{bar})$ & $\left\langle N_{\mathrm{Ar}}\right\rangle$ & $\left\langle N_{\mathrm{Kr}}\right\rangle$ & $\left\langle N_{\mathrm{ArKr}}\right\rangle$ \\
\hline $\mathrm{Ar}$ & - & - & 113.5 & 2.50 & 7000 & - & - \\
\hline $\mathrm{ArKr}$ & 3.0 & $37(6)$ & 125 & 0.65 & 102 & 414 & 109 \\
$\mathrm{ArKr}$ & 5.0 & $35(5)$ & 118 & 2.50 & 5600 & 22500 & 6100 \\
$\mathrm{ArKr}$ & 5.0 & $47(6)$ & 118 & 1.50 & 1600 & 6500 & 1800 \\
\hline $\mathrm{Kr}$ & 100 & 100 & 123 & 0.76 & - & 700 & - \\
\hline
\end{tabular}

${ }^{a}$ Ref. [37] 
conversion was performed based on a set of helium (He 1s) spectra measured with the same settings of the instrument over a range of kinetic energies. The photoelectron spectra in all three panels show the spin-orbit split Ar $3 \mathrm{p}_{1 / 2}$ and $\mathrm{Ar} 3 \mathrm{p}_{3 / 2}$ monomer bands at a binding energy of around $15.8 \mathrm{eV}$. The $\operatorname{Kr} 4 \mathrm{p}_{3 / 2,1 / 2}$ monomer contributions are only visible in panel a) at 14.0 $\mathrm{eV}$ and $14.6 \mathrm{eV}$ binding energy, respectively. Panel a) further shows a sharp feature at a binding energy of about $15.6 \mathrm{eV}$. This feature stems from fast electrons generated by higher harmonic light formed in the undulator and can be neglected.

In order to obtain the $\mathrm{Kr}$ content in the final clusters $\left(\mathrm{Kr}_{\mathrm{cl}}\right)$, which can differ substantially from the initial gas mixture, we determined the area under the electron spectra of the Ar 3p and Kr 4p cluster bands, respectively. This ratio is then corrected by the atomic cross sections for the Ar $3 \mathrm{p}(30.7 \mathrm{Mb}$ ) and $\mathrm{Kr} 4 \mathrm{p}(42.9 \mathrm{Mb})$ bands at $\mathrm{h} \nu=16.2 \mathrm{eV}$ [54]. The result is given in Tab. 1.

All inner valence spectra were recorded at an ionization energy of $32.0 \mathrm{eV}$. The total acquisition times for the inner valence spectra were between 600 and $1400 \mathrm{~s}$. For each single bunch, all electron arrival times were recorded and stored together with a unique identifier. Every time we record exactly two electrons within the time period of one single bunch (800 ns), we call this a two electron coincidence. The total count rate is kept below 1/30 of the bunch repetition rate. This way we can keep the rate of random coincidences (coincidences, in which the two electrons stem from two uncorrelated ionization events) much below the rate of the true coincidences (coincidences in which the two generated electrons are correlated with each other). The 
resulting coincidence spectra can be depicted in a two dimensional map. A main advantage of this method compared to conventional, non-coincident spectroscopy, is that electrons can be identified that have the same energy as electrons from other processes, even if the other processes have a higher efficiency. In our case we can identify very slow autoionization electrons above a background of slow electrons stemming from intracluster scattering processes. A detailed description of this method can be found in [50, 55].

In order to obtain the efficiency of an autoionization process under investigation, we can relate the number of recorded photoionization electrons to the number of recorded autoionization electrons. Each value has to be corrected by the detection probability of the respective electrons. This, again, is instrument dependent and can additional vary for electrons of different kinetic energy. To determine the detection probability we have recorded Xe $4 \mathrm{~d}$ photoelectrons in coincidence with their $\mathrm{N}_{4,5} \mathrm{OO}$ Auger electrons, which have known branching ratios and kinetic energies. A detailed description of this method is given in [56]. 


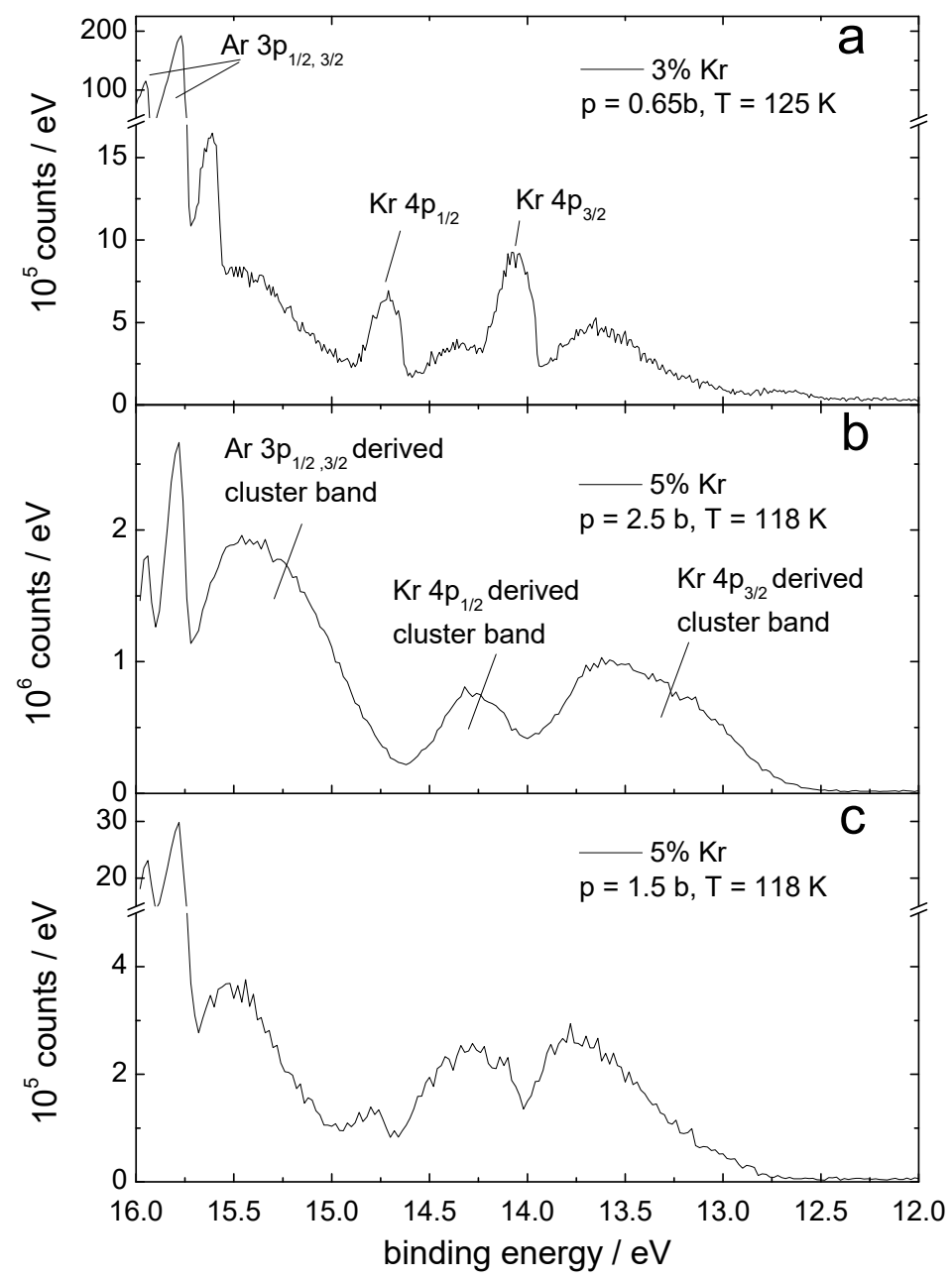

Figure 3: Outer valence photoelectron spectra of mixed ArKr clusters, ionized at a photon energy of $\mathrm{h} \nu=16.2 \mathrm{eV}$. Labels in the individual panels denote amount of $\mathrm{Kr}$ in the initial gas mixture, and other expansion conditions, see Tab. 1. The feature visible in panel a) at $15.6 \mathrm{eV}$ is an experimental artifact stemming from electrons emitted by higher order synchrotron radiation. Also included are indicators for the positions of the Ar $3 p$ and $\mathrm{Kr}$ $4 \mathrm{p}$ monomer lines in panel a) and for the $\mathrm{Ar}_{16} \mathrm{p}$ and $\mathrm{Kr} 4 \mathrm{p}$ derived cluster bands in panel b). 


\section{Computational Details}

The computations of the ETMD spectra were performed with the program HARDRoC [57], which decomposes the entire system into triples. Here, the term 'triple' designates a set of three atoms (one Ar, two $\mathrm{Kr}$ ) at given positions in the cluster, including the assignment of the roles $X_{\mathrm{in}}, X_{\mathrm{D}}$ and $X_{\mathrm{E}}$ in the ETMD process (see Fig. 2). Each set of three atoms therefore gives rise to six 'triples', two of which (the ones with $X_{\text {in }}=\operatorname{Ar}$ ) are of interest here. Generally, each triple is characterized by different electron transfer distances $Q$, energy transfer distances $R$ and angles $\alpha$. The ETMD electron energies and decay widths are then simulated for each triple using properties of the subsystems and units. These properties have been determined experimentally (see Table 2), but to the best of our knowledge, experimental transition dipole moments are not available for the ArKr dimer. The latter were therefore calculated with the Kramers-restricted configuration interaction method in the 2012 release of the relativistic quantum chemistry program package DIRAC [58-61], using dual basis sets for argon (17s12p4d4f2g) and krypton $(21 s 19 p 15 d 4 f 3 g)$. Because the transition dipole moments depend on the interatomic distance in subsystem 1, they were calculated for several distances and the set was fitted to the expression $\left\langle\tilde{D}_{j}\right\rangle=b \exp (-\chi Q)+c$, which then was evaluated for each triple. Values at the equilibrium distance of $3.90 \AA$ are given in Table 2.

The van der Waals radii used for the construction of the structures are $r_{A r}=1.88 \AA$ and $r_{K r}=2.02 \AA$ [63]. 
Table 2: Experimental and calculated values for the parameters used for the simulation of the ETMD electron spectra. The single ionization potentials were determined experimentally for ArKr clusters [55]. Ionization cross sections are from literature data for gaseous atoms. Here, $\sigma\left(\omega_{j}\right)$ designates the full ionization cross section of atomic $\mathrm{Kr}$ at the (virtual) photon energy corresponding to the filling of an $\mathrm{Ar} 3 \mathrm{~s}$ vacancy by a $\mathrm{Kr} 4 \mathrm{p}_{j}$ electron, $\omega_{j}=S I P\left(X_{\mathrm{in}}\right)-S I P\left(X_{\mathrm{D}, \mathrm{j}}\right)$. The cross sections for creation of vacancies in the two different $\mathrm{Kr} 4 \mathrm{p}$ fine-structure states at the site $X_{E}$ were derived by partitioning each $\sigma\left(\omega_{j}\right)$, taking into account the experimentally observed ionization cross section ratio $\sigma\left(\operatorname{Kr} 4 \mathrm{p}_{3 / 2}\right) / \sigma\left(\operatorname{Kr} 4 \mathrm{p}_{1 / 2}\right)$ (last line).

\begin{tabular}{lrc}
\hline property & value \\
\hline $\mathrm{SIP}(\operatorname{Ar} 3 \mathrm{~s})$ & $28.80 \mathrm{eV}$ & \\
$\mathrm{SIP}(\operatorname{Ar} 3 \mathrm{p})$ & $15.37 \mathrm{eV}$ & \\
$\mathrm{SIP}\left(\mathrm{Kr} 4 \mathrm{p}_{1 / 2}\right)$ & $13.40 \mathrm{eV}$ & \\
$\operatorname{SIP}\left(\operatorname{Kr} 4 \mathrm{p}_{3 / 2}\right)$ & $15.37 \mathrm{eV}$ & \\
\hline $\operatorname{SIP}\left(\operatorname{Ar} 3 \mathrm{p}_{\text {nrel }}\right)$ & $13.68 \mathrm{eV}$ & \\
$\operatorname{SIP}\left(\operatorname{Kr} 4 \mathrm{p}_{\text {nrel }}\right)$ & $1.365 \cdot 10^{-2} \mathrm{Db}$ & \\
\hline$\langle 3 / 2|\tilde{x}| 1 / 2\rangle$ & $19.927 \cdot 10^{-2} \mathrm{Db}$ & \\
$\langle 1 / 2|\tilde{z}| 1 / 2\rangle$ & $-0.374 \cdot 10^{-2} \mathrm{Db}$ & \\
$\langle-1 / 2|\tilde{x}| 1 / 2\rangle$ & $22.568 \cdot 10^{-2} \mathrm{Db}$ & \\
$\langle 1 / 2|\tilde{z}| 1 / 2\rangle$ & $-3.619 \cdot 10^{-2} \mathrm{Db}$ & \\
$\langle-1 / 2|\tilde{x}| 1 / 2\rangle$ & $40.16 \mathrm{Mb}$ & {$[54]$} \\
\hline$\sigma\left(\omega_{3 / 2}\right)$ & $36.51 \mathrm{Mb}$ & {$[54]$} \\
$\sigma\left(\omega_{1 / 2}\right)$ & \multicolumn{3}{|c}{1.77} & {$[62]$} \\
$\sigma_{3 / 2} / \sigma_{1 / 2}$ & & \\
\hline
\end{tabular}




\section{ETMD3 in a trimer}

In order to understand the ETMD electron spectra of larger clusters, it is helpful to start with the properties of triples of atoms, from which larger clusters can be composed. The most important question is whether a decay channel is open at all or not. Therefore, we determined the channel opening distances $d_{\text {open }}$ of the four different channels $\beta$, and the hypothetic non-relativistic channel, by evaluating Eq. (4) for a kinetic energy of the secondary electron of zero. The results are shown in Table 3.

Table 3: Channel opening distances $d_{\text {open }}$ between the electron donating $X_{D}$ and the electron emitting unit $X_{E}$ for the different decay channels and the estimated, non-relativistic counterpart.

\begin{tabular}{lr}
\hline channel $\beta$ & $d_{\text {open }}$ \\
\hline ArKr $4 \mathrm{p}_{3 / 2}^{-1} \mathrm{Kr} 4 \mathrm{p}_{3 / 2}^{-1}$ & $7.20 \AA$ \\
$\operatorname{ArKr} 4 \mathrm{p}_{3 / 2}^{-1} \mathrm{Kr} 4 \mathrm{p}_{1 / 2}^{-1}$ & $12.52 \AA$ \\
$\operatorname{ArKr} 4 \mathrm{p}_{1 / 2}^{-1} \mathrm{Kr} 4 \mathrm{p}_{3 / 2}^{-1}$ & $12.52 \AA$ \\
$\operatorname{ArKr} 4 \mathrm{p}_{1 / 2}^{-1} \mathrm{Kr} 4 \mathrm{p}_{1 / 2}^{-1}$ & $48.00 \AA$ \\
\hline $\operatorname{ArKr} 4 \mathrm{p}_{\text {nrel }}^{-1} \mathrm{Kr} 4 \mathrm{p}_{\text {nrel }}^{-1}$ & $9.35 \AA$ \\
\hline
\end{tabular}

The ArKr $4 \mathrm{p}_{1 / 2}^{-1} \mathrm{Kr} 4 \mathrm{p}_{1 / 2}^{-1}$ channel is unlikely to be visible in the spectrum at all, and the ArKr $4 \mathrm{p}_{3 / 2}^{-1} \mathrm{Kr} 4 \mathrm{p}_{1 / 2}^{-1}$ and ArKr $4 \mathrm{p}_{1 / 2}^{-1} \mathrm{Kr} 4 \mathrm{p}_{3 / 2}^{-1}$ channels only make up for a minor part of the spectrum due to the $R^{-6}$-behaviour of the decay width.

In Jacobi coordinates, the distance between the two ionized units in the 
final state $D$ is given by:

$$
d=\sqrt{R^{2}-2 R Q \cos \alpha+Q^{2}}
$$

Hence, the channel opening angle $\alpha_{\text {open }}$ and the channel opening energy transfer distance read

$$
\begin{aligned}
& R_{\text {open }}=Q \cos \alpha_{\text {open }}+\sqrt{Q^{2}\left(\cos ^{2} \alpha_{\text {open }}-1\right)+d^{2}} \\
& \alpha_{\text {open }}=\arccos \left(\frac{R_{\text {open }}^{2}+Q^{2}-d^{2}}{2 R_{\text {open }} Q}\right) .
\end{aligned}
$$

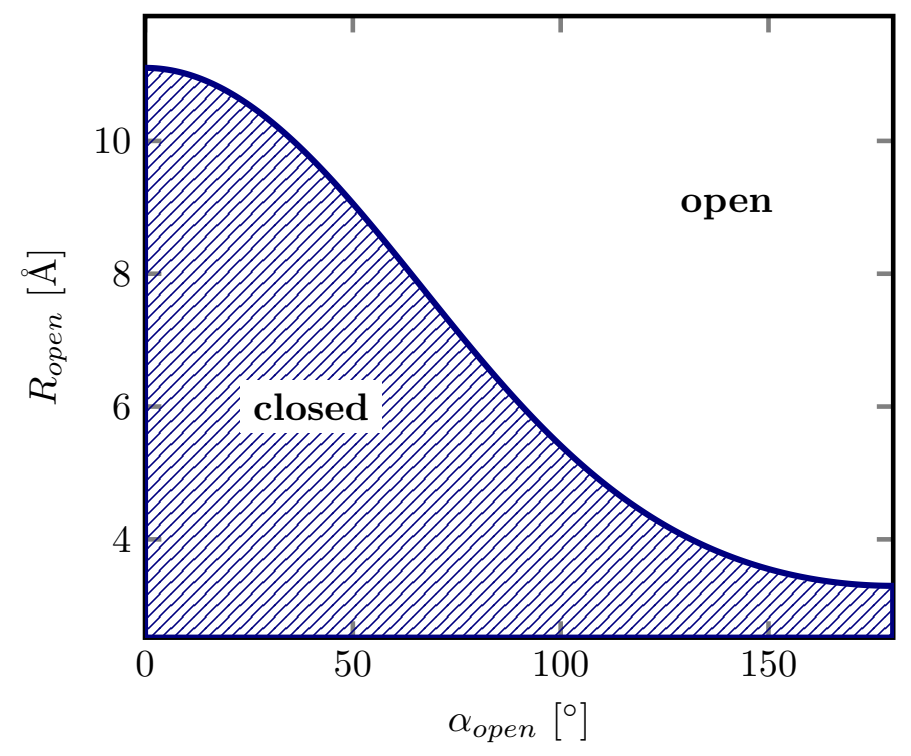

Figure 4: Channel opening coordinates for the ArKr $4 \mathrm{p}_{3 / 2}^{-1} \mathrm{Kr} 4 \mathrm{p}_{3 / 2}^{-1}$ decay channel evaluated with a fixed $Q=3.90 \AA$ and $d_{\text {open }}=7.20 \AA$ (see Eq. (9)). The emitter channel opening distance $R$ is large for small angles $\alpha$ and decreases with increasing angle to a distance of $3.3 \AA$.

253 For a constant value of the electron transfer distance $Q=3.90 \AA$, the 254 interplay between the energy transfer distance $R$ and the angle $\alpha$ are dis255 played for the $\mathrm{ArKr}_{2}$ trimer in Figure 4. At small angles the channel opening 
distance $R_{\text {open }}$ is large and then decreases with the opening of the trimer angle towards $180^{\circ}$. The smallest channel opening distance is even below the equilibrium interatomic distance of the $\operatorname{ArKr}$ dimer $\left(R_{e q}(\operatorname{ArKr})=3.90 \AA\right)$. Hence, for large angles the ETMD channel is open even for direct neighbours and would also be open in the corresponding trimer. These findings support and generalize the theoretical investigation of the ArKr trimer in [40].

Throughout the following discussion of the geometry dependence of the decay width, we choose one krypton atom as the electron donor and the other atom as the electron emitting species. In reality both atoms can be donor als well as emitter without an influence on the energy of the resulting final state, that is the two cases are experimentally indistinguishable. If the distances of both to the initially ionized atom are equal, the two alternatives contribute the same amount to the total decay rate. If one of the atoms ionized in the final state is significantly further away, its probability in donating the electron, which is filling the vacancy, decreases exponentially and can hence be neglected. For simplicity we skip this discussion for trimers, we will return to it when discussing ETMD3 in larger clusters.

From Eq. (6) the $R$ dependence of $\Gamma$ is read as $\Gamma \propto R^{-6}$, corresponding to an energy transfer mainly being caused by a dipole-dipole interaction. The dependence on $Q$ is implicitely included in the transition dipole moments. These depend on the overlap between the two atoms participating in the electron transfer, which decreases exponentially with $Q$.

The angular part of equation (6) can be reformulated to yield:

$$
\Gamma_{i} \propto \sum_{M_{i n, D^{\prime}}} 2\left[\left|\left\langle\tilde{D}_{z}\left(M_{i n, D^{\prime}}\right)\right\rangle\right|^{2}\left(1+\cos ^{2} \alpha\right)+\left|\left\langle\tilde{D}_{x}\left(M_{i n, D^{\prime}}\right)\right\rangle\right|^{2}\left(2+\sin ^{2} \alpha\right)\right] .
$$


We now discuss the influence of the geometrical properties of a triple, and in particular of its opening angle $\alpha$, on the decay width. In Figure 5, we show the ETMD3 decay width as a function of $\alpha$. From Eq. (10), it is clear that the two orientations of the transition dipole matrix element influence $\Gamma_{i}$ in a different way, and the two factors are shown separately in the upper panel of the Figure. The two curves are mirror images of each other with respect to a horizontal drawn at $\Gamma=4$.

We now consider a model with two contributions to the decay width: one with $\left\langle\tilde{D}_{z}\right\rangle=0$ and $\left\langle\tilde{D}_{x}\right\rangle \neq 0$ and the other with $\left\langle\tilde{D}_{z}\right\rangle \neq 0$ and $\left\langle\tilde{D}_{x}\right\rangle=0$. This corresponds e.g. to the decay width with $X_{D}$ being $\operatorname{Kr} 4 \mathrm{p}_{1 / 2}^{-1}$. Due to the larger orbital overlap for orbitals along the bonding axis $(\tilde{z})$ compared to the orbital overlap of orbitals perpendicular to it $(\tilde{x}),\left|\left\langle\tilde{D}_{z}\right\rangle\right|^{2}$ is typically about one order of magnitude larger than $\left|\left\langle\tilde{D}_{x}\right\rangle\right|^{2}$, but the exact figure can vary. We therefore define $q=\left|\left\langle\tilde{D}_{x}\right\rangle\right|^{2} /\left|\left\langle\tilde{D}_{z}\right\rangle\right|^{2}$, with $q \leq 1$. The angular dependence then reads:

$$
\Gamma_{i} \propto 4 q+2(q-1) \sin ^{2} \alpha+4
$$

and is illustrated in the lower panel of Figure 5. It is an oscillating function with maxima at even multiples of $\pi / 2$ and minima at uneven multiples at $\pi / 2$, unless $q=1$. In the case of $q$ approaching zero, the angular part of the decay width approaches a shifted $2 \cos ^{2}(\alpha)$-curve with maxima at even multiples of $\pi / 2$ and minima at uneven multiples of $\pi / 2$, with values between 4 and 2 . Therefore, for typical values of $q$, the energy transfer to an atom on the same axis $(\alpha=0, \pi)$, corresponding to a linear arrangement, is preferred.

Combining both, the view on the energetic accessibility of the decay channels and the decay widths, results in the pictures shown in Figure 6. Here the 
geometry dependence of the decay width for the case of the three relevant $\mathrm{ArKr}_{2}$ channels is displayed. The numbers were obtained by HARDRoC using the asymptotic formula in Eq. (6) for different combinations of $R$ and $\alpha$. Here, the charge transfer distance $Q=3.90 \AA$ is chosen to be equal to the internuclear distance of the neutral ArKr dimer. The plots display the channel closing for very small $\mathrm{Kr}-\mathrm{Kr}$ distances and the $R^{-6}$ behaviour, as well as the expected angle dependence explicitly shown in Figure 5. However, the angle dependence is not easily recognized due to the channel closing at small distances.

The decay widths of the three different decay channels shown in Figure 6 can be summed up to yield the total decay width of the $\mathrm{ArKr}_{2}$ trimer, as illustrated in Figure 7. The upper panel shows the full range of calculated decay widths, while in the lower panel we zoom into the upper panel and show the decay widths for $R \geq 6.00 \AA$. Also here, the $R^{-6}$ dependence is observed and the dependence on the angle $\alpha$ is better visible than in Figure 6. However, it is not fully visible as the $\operatorname{ArKr} 4 \mathrm{p}_{3 / 2}^{-1} \mathrm{Kr} 4 \mathrm{p}_{3 / 2}^{-1}$ channel is closed at small angles due to the shorter interatomic distance between the two krypton atoms. Around $R=9 \AA$ and at high angles in the lower panel, the decay width shows a rapid increase. These "spikes" are caused by the channel opening of the ArKr $4 \mathrm{p}_{3 / 2}^{-1} \mathrm{Kr} 4 \mathrm{p}_{1 / 2}^{-1}$ and $\operatorname{ArKr} 4 \mathrm{p}_{1 / 2}^{-1} \mathrm{Kr} 4 \mathrm{p}_{3 / 2}^{-1}$ channels. The plots of the decay width clearly show the dominance of the ArKr $4 \mathrm{p}_{3 / 2}^{-1} \mathrm{Kr}$ $4 \mathrm{p}_{3 / 2}^{-1}$ channel over the other ETMD3 channels. 

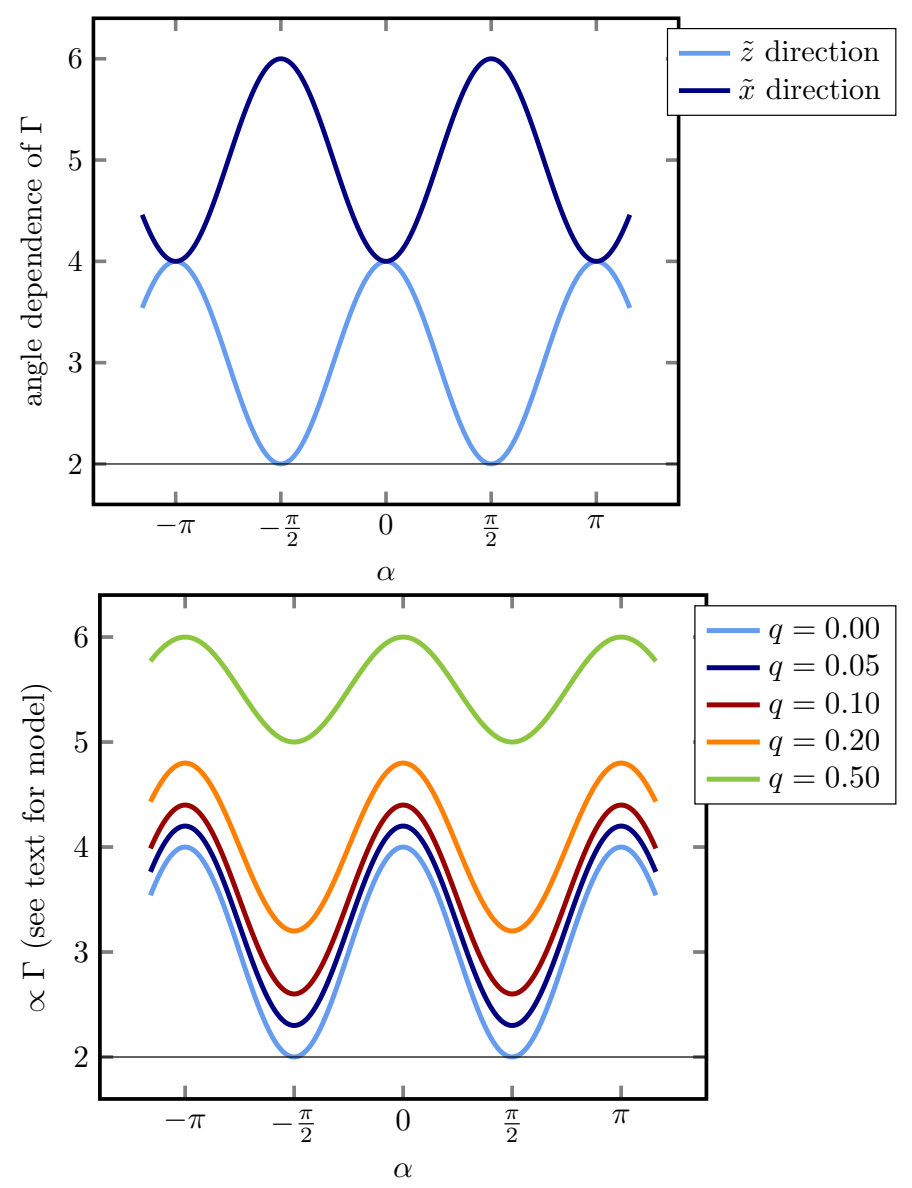

Figure 5: Upper panel: ETMD3 decay width vs. trimer angle $\alpha$. Values are shown separately for the contributions of the two different dipole transition matrix elements of the electron transfer step, corresponding to an orientation of the dipole along the internuclear axis of subsystem $S_{1}$ ( $\tilde{z}$-direction, light curve) or perpendicular to it ( $\tilde{x}$-direction, dark curve). Lower panel: ETMD3 decay width vs. trimer angle $\alpha$, for electron transfer with simultaneous contributions of both transition dipoles. The curves are parametrized by the ratio of the respective dipole moments, $q=\left|\left\langle\tilde{D}_{x}\right\rangle\right|^{2} /\left|\left\langle\tilde{D}_{z}\right\rangle\right|^{2}$. See Eq. 6 and Fig. 2. 

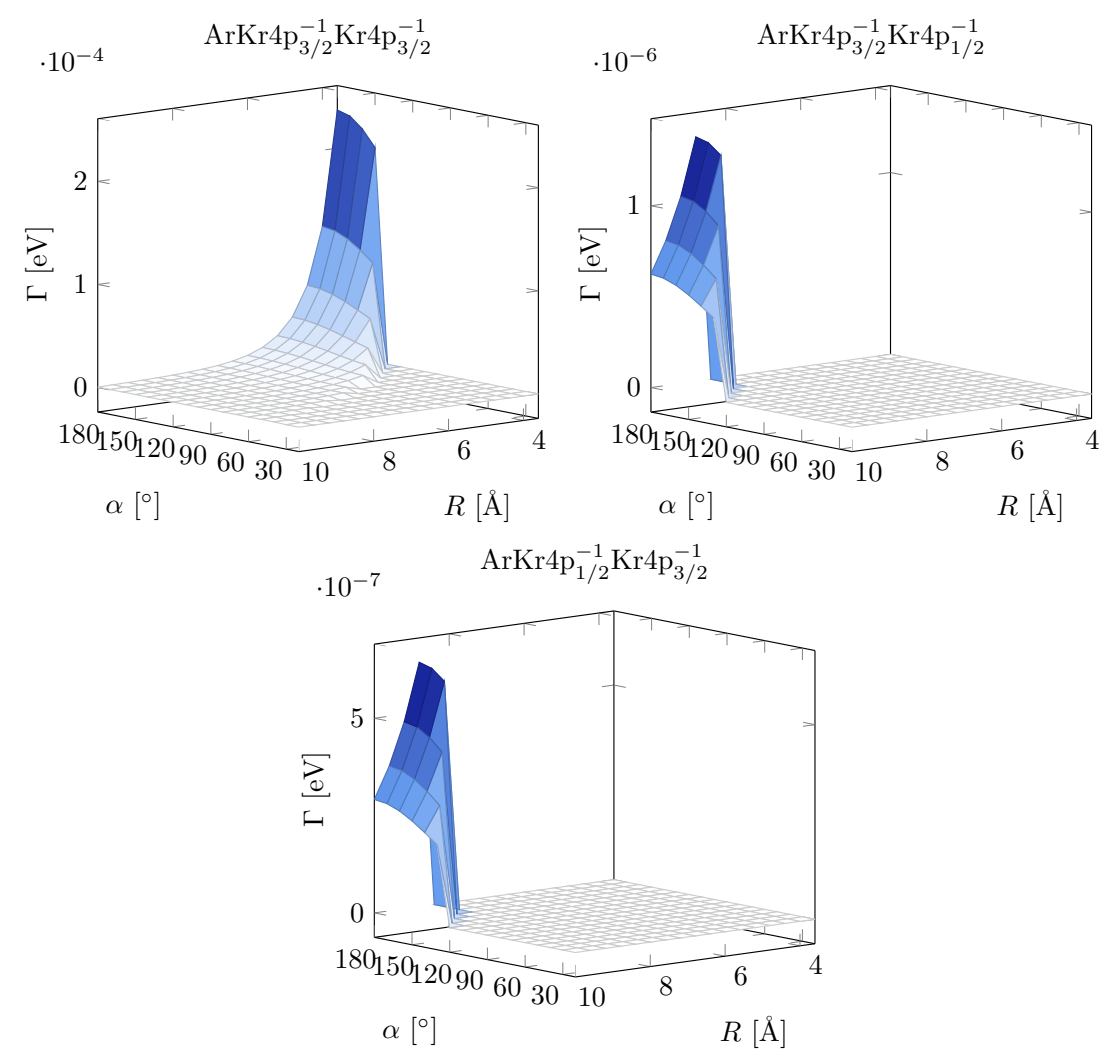

Figure 6: ETMD3 partial decay width $\Gamma$ vs. trimer angle $\alpha$ and energy transfer distance $R$ for an $\mathrm{ArKr}_{2}$ trimer with a fixed donor atom. The three open electronic decay channels are shown separately. 

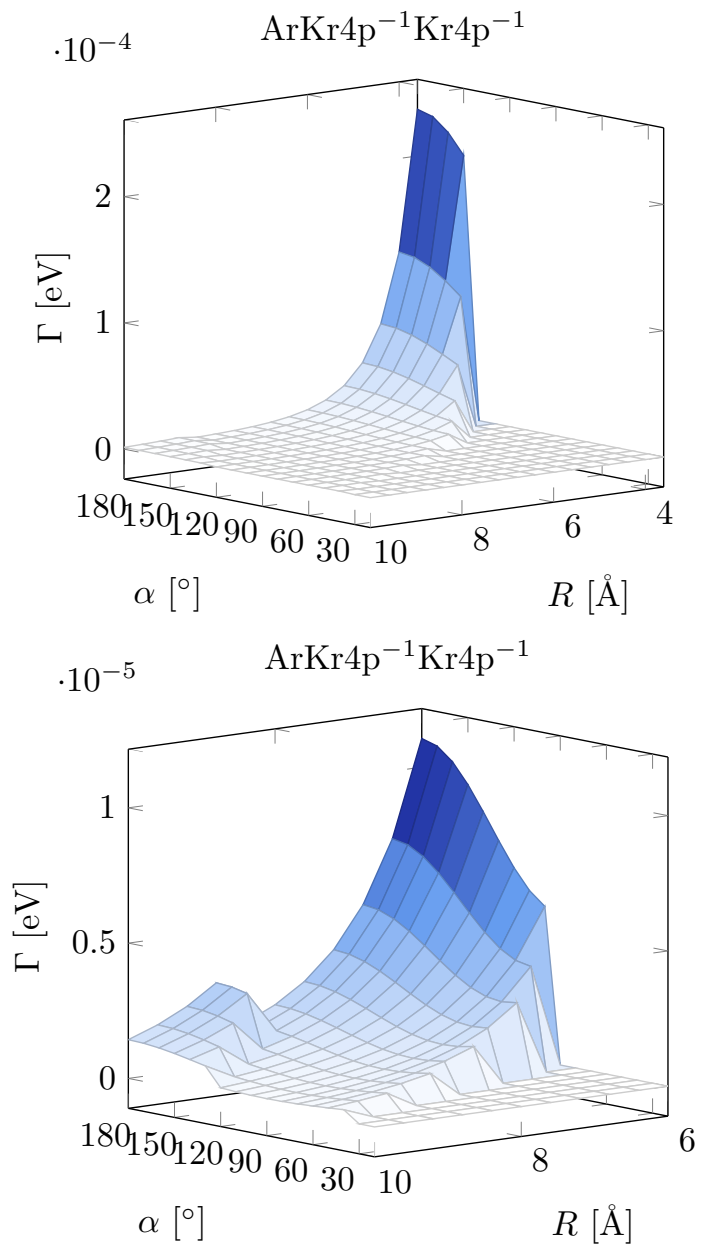

Figure 7: ETMD3 total decay width (sum over the decay widths of the three open channels, illustrated separately in Figure 6). The opening of channels at different angles $\alpha$ and internuclear distances $R$ is observed at the spikes. 


\section{ETMD in ArKr Clusters}

In a cluster consisting of more than three atoms, the ETMD3 spectra pertaining to several combinations of three atoms (triples) overlap. Additionally, each set of one argon and two krypton atoms makes up for two triples, in which the role of the electron donor and emitter is interchanged between the two krypton atoms. Charge stabilization of the ionized states in clusters is taken care of by using experimental ionization energies of mixed ArKr clusters of a size appropriate for comparison with our experiments. By that, a single ionization energy is used for each type of atom and therefore, energy differences between core and shell positions, or different positions within one layer, are not taken into account. This will lead to a further broadening of the experimental spectrum, compared to the spectrum calculated in this approach.

Most of the available experimental secondary electron spectra of ArKr clusters are from clusters with mean sizes of $\langle N\rangle<2000$, we therefore study cluster structures based on an icosahedral structure, which is most common for rare gas clusters of this size. One cluster structure was created as an idealized icosahedral structure with a krypton core of four shells (147 atoms) surrounded by a complete shell of argon atoms (see the left structure in Figure 8). The interatomic distances were calculated using the van-der-Waals radii of the atoms.

Core-shell structures in mixed rare gas clusters are energetically favoured due to differences in the respective cohesive energies of their components, which are $0.02 \mathrm{eV}, 0.08 \mathrm{eV}, 0.116 \mathrm{eV}$ and $0.16 \mathrm{eV}$ for solid neon, argon, krypton and xenon, respectively [64]. Experimentally, core-shell structures have 

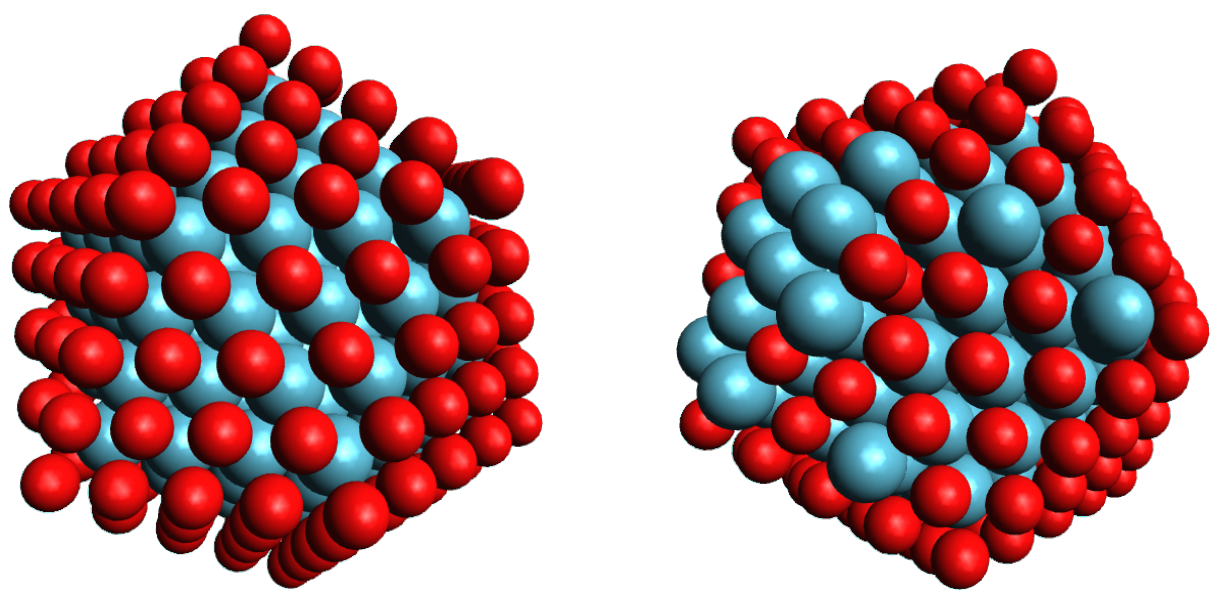

Figure 8: Structures of the investigated ArKr clusters investigated theoretically. Both contain 309 atoms (147 krypton and 162 argon atoms). In the left panel the cluster has an icosahedral core-shell structure with a krypton core surrounded by one layer of argon atoms. The cluster in the right panel has an overall icosahedral structure with a krypton core surrounded by argon atoms in which 14 argon and krypton atoms are randomly interchanged.

been shown to be dominant for NeAr and ArXe clusters [65, 66]. However, in the case of ArKr, where the cohesive energies are very similar, a core-shell structure can not necessarily be assumed, since no clear interface layer of krypton atoms could be detected experimentally [67], which is in contrast to the corresponding experiment of ArXe clusters [66]. Some diffusion of Ar into a $\mathrm{Kr}$ core has also been found in molecular dynamics simulations [68]. Theoretical studies have shown that, if krypton atoms are found outside the core region, they occupy high coordination sites in either surface or edge positions [69]. Therefore, we constructed a second cluster structure by starting from the idealized icosahedral cluster. Then, the positions of 14 pairs of argon and krypton atoms were interchanged randomly, such that the kryp- 
ton atoms were located at high coordination surface positions. The obtained structure was then optimized using a universal force field implemented in the program Avogadro (version 1.1.0) [70, 71] (see the right cluster structure in Figure 8). This attempt might lead to a local rather than a global minimum energy structure, but does allow to study the influence of argon atoms in the sub-surface region.

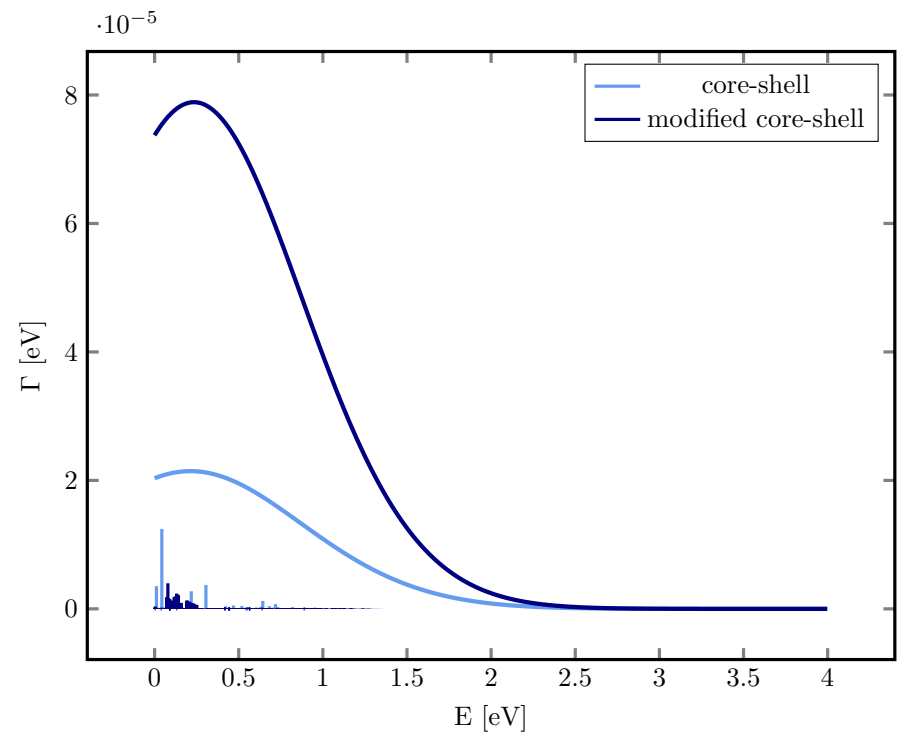

Figure 9: ETMD spectrum of an ArKr cluster consisting of a $137 \mathrm{Kr}$ atoms icosahedral core surrounded by a complete shell of Ar atoms (309 atoms in total). The ETMD electron spectrum is dominated by several peaks close to $0 \mathrm{eV}$ leading to a maximum around $0.2 \mathrm{eV}$ and a tail extending up to $2 \mathrm{eV}$. The $\mathrm{ArKr} 4 \mathrm{p}_{3 / 2}^{-1} \mathrm{Kr} 4 \mathrm{p}_{3 / 2}^{-1}$ channel is responsible for $89 \%$ of the total decay width.

Since other electronic decay mechanims are energetically not accessible in these clusters, we only simulated the ETMD3 spectrum, with the result shown in Figure 9.

The spectrum of the idealized cluster is composed out of several smaller 
peaks which have been convoluted with Gaussians with a width of $600 \mathrm{meV}$ to take care of vibrational effects and to guide the eye of the reader. The resulting signal is very broad with a maximum close to $0 \mathrm{eV}$ and decreases up to approximately $2 \mathrm{eV}$. A further fine structure is not visible. This is in very good agreement with the experimental spectra shown in Ref. [37], and in this work. Further analysis of the spectrum shows that the unresolved peaks between ca. $0.5 \mathrm{eV}$ and $1 \mathrm{eV}$ correspond to ETMD3 with nonnearest neighbours. The ArKr $4 \mathrm{p}_{3 / 2}^{-1} \mathrm{Kr}_{4} \mathrm{p}_{3 / 2}^{-1}$ channel is dominant in the cluster, and is responsible for $89 \%$ of the total decay width per argon atom.

In Figure 10, the angle distribution of the triples that are responsible for the decay width, is shown for the ArKr $4 \mathrm{p}_{3 / 2}^{-1} \mathrm{Kr}_{4} \mathrm{p}_{3 / 2}^{-1}$ channel. It shows that triples with angles higher than $110^{\circ}$ do not contribute to the ETMD3 spectrum of the core-shell cluster. This result has a simple geometrical explanation: As always the initially ionized Ar atom is located on the surface of the cluster, approx. $110^{\circ}$ is the largest value of the trimer opening angle that occurs.

Most triples that can be constructed from the cluster atoms have angles $\alpha$ between $70^{\circ}$ and $100^{\circ}$. Hence, they pertain to the angle interval with rather low decay width (see Fig. 5). However, in the idealized core-shell cluster structure, these angles allow a decay with nearest neighbours; and in this situation the $R^{-6}$-behaviour of the decay width leads to an advantage of these triples over the other ones with smaller angles.

The ETMD spectrum using the non-relativistic approach [28] was simulated as well (not shown). After convolution, the spectrum and the decay width per argon atom are almost identical to their relativistic couterparts. 


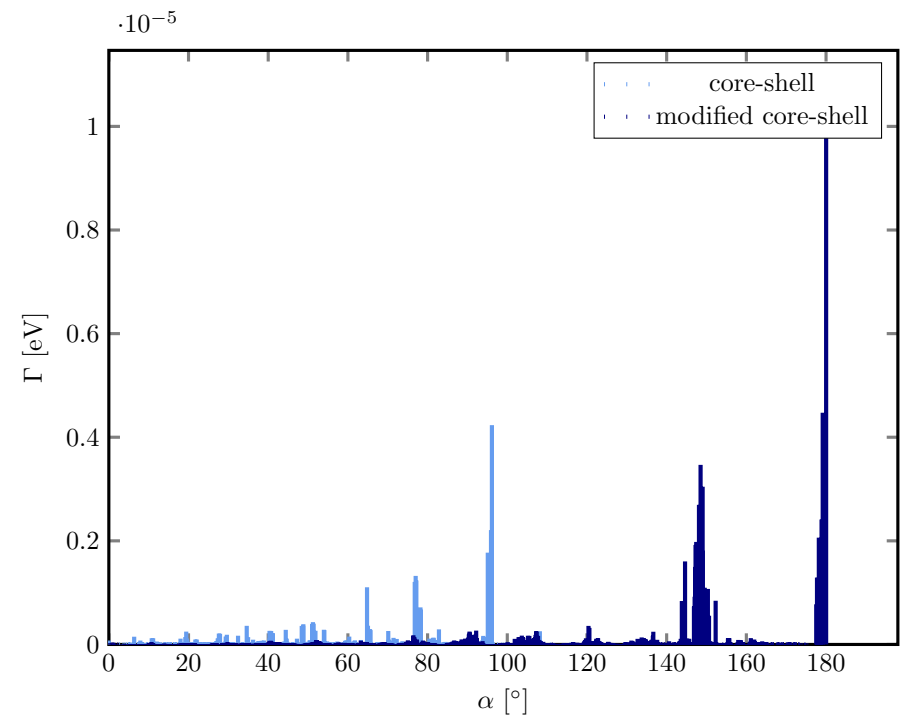

Figure 10: Angle dependence of the decay width of the dominant $\operatorname{ArKr} 4 \mathrm{p}_{3 / 2}^{-1} \mathrm{Kr} 4 \mathrm{p}_{3 / 2}^{-1}$ channel in an icosahedral ArKr cluster consisting of 309 atoms (147 krypton atoms and 162 argon atoms). The largest contribution to the total decay width results from trimers with angles between $60^{\circ}$ and $100^{\circ}$, even though the decay width of a single trimer shows a minimum at an angle $\alpha=90^{\circ}$. Larger angles are rarely found in core-shell cluster structures and triples with smaller angles are (in this model) characterized by large energy transfer distances and hence lower decay widths. See text for further discussion.

The decay channel is not open for as many triples as in the relativistic case, however, when it is open, the decay width is not split over different channels and is therefore higher than the decay width of the $\operatorname{ArKr} 4 \mathrm{p}_{3 / 2}^{-1} \mathrm{Kr} 4 \mathrm{p}_{3 / 2}^{-1}$ channel.

The modified icosahedral cluster structure shows a very similar ETMD3 spectrum (see Figure 9). Also here, the ArKr $4 \mathrm{p}_{3 / 2}^{-1} \mathrm{Kr} 4 \mathrm{p}_{3 / 2}^{-1}$ channel is dominant. However, the decay width is higher and the spectrum is broader. Since the overall number of triples is exactly the same as in the idealized cluster 
structure, this difference is obviously caused by the rearrangement of the atoms. Due to the presence of argon atoms in the sub-surface region, the same interatomic distance $d$ between two krypton atoms can be achieved in triples with smaller distances $Q$ and $R$, thereby also having a larger opening angle $\alpha$. This can be seen well from the angle distribution of the decay width in Figure 10. Since the decay widths for constant $Q$ and $R$ are largest at $\alpha=180^{\circ}$, the total decay width of the cluster is affected by this structural change. This effect on the decay width per initially ionized argon atom is quantified in Table 4.

Table 4: Average ETMD3 decay width $\Gamma$ and lifetime $\tau$ of the two cluster structures. For comparison, values for one triple and for a full trimer, both in the geometrically most favourable configuration, are shown.

\begin{tabular}{lrr}
\hline & $\Gamma\left[10^{-5} \mathrm{eV}\right]$ & $\tau[\mathrm{fs}]$ \\
\hline core-shell & 3.56 & 18.49 \\
modified core-shell & 12.70 & 5.18 \\
triple $\alpha=180^{\circ}, R=4.0 \AA$ & 12.60 & 5.22 \\
trimer $\alpha=180^{\circ}, R=4.0 \AA$ & 22.84 & 2.88 \\
\hline
\end{tabular}

All lifetimes, both for the triple and the trimer as well as for the two cluster structures, are almost six orders of magnitude smaller than the lifetime due to radiative decay of an atomic $\mathrm{Ar} 3 \mathrm{~s}^{-1}$ vacancy, which is $4.68 \mathrm{~ns}$ [72]. Hence, according to our simulations ETMD3 is the dominant decay process in ArKr clusters after 3s ionization. (Experimental results underpinning this assertion will be presented below.) The decay width of the modified core-shell structure is almost four times larger than the decay width of the idealized 
core-shell cluster structure.

This can be attributed to two geometrical factors: Many of the triples involved in the decay of the modified core-shell system have large opening angles $\alpha$, up to (almost) $180^{\circ}$ and are additionally characterized by short energy transfer and charge transfer distances, $R$ and $Q$. The first condition leads to an increase of the decay width by about a factor of two, and the effect of the second condition is comparable, as the two triples with interchanged roles of donor and emitter contribute with almost the same magnitude. At the same time, the average decay width per initially ionized argon atom is approximately the same as for a specific triple structure, but is significantly smaller than the decay width of a trimer with short $R$ and $Q$. This can be explained by the occurence of a multitude of other triples with less favourable geometric parameters, which decreases the average decay width.

\section{Experimental results}

The experimental electron emission spectrum of Ar $3 \mathrm{~s}$ ionized states in Ar-Kr clusters has been presented by some of the authors [37]. In that work, coexpansion of Ar gas with a $5 \%$ admixture of $\mathrm{Kr}$ was used for cluster production, leading to clusters with a $\mathrm{Kr}$ fraction of about $40 \%$ (Tab. 1). In order to investigate the dependence of this signal on the cluster parameters, we have repeated the experiment with different gas mixtures and expansion conditions. The electron-electron coincidence spectrum of clusters from Ar gas with a $3 \%$ admixture of $\mathrm{Kr}$ is shown in Fig. 11. Here, as in some of our earlier work [41, 50,73], we show a colour coded map of the intensity distribution of electron pairs vs. the two electron energies, with $e_{1}$ designat- 


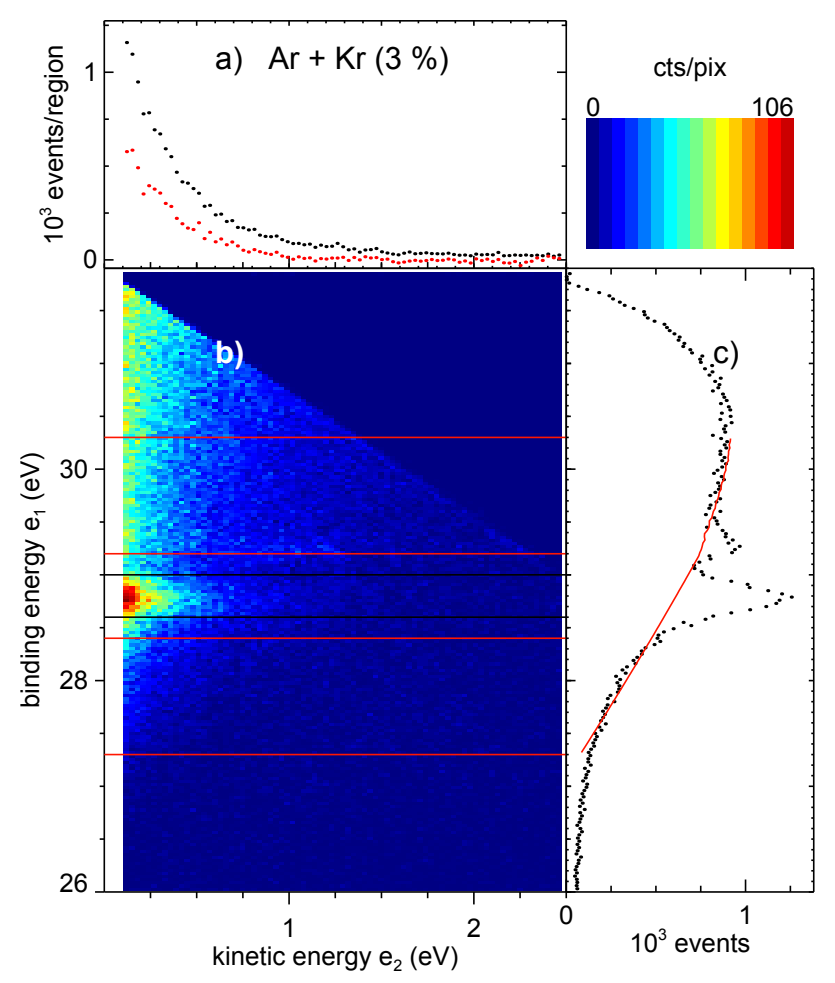

Figure 11: Photon excited electron-electron coincidence spectrum of mixed Ar-Kr clusters ( $3 \% \mathrm{Kr}$ in the initial gas mixture) in the region of Ar inner valence electrons. (b): Colorcoded map of coincident electron pairs, with the electron of higher kinetic named $e_{1}$. The energy of $e_{1}$ is given as binding energy, using the photon energy of $h \nu=32 \mathrm{eV}$. (c): Energy spectrum of primary electrons $e_{1}$, irrespective of the energy of the secondary electron (summation of the coincidence map along horizontal lines). (a): Energy spectrum of all secondary (ETMD) electrons $e_{2}$ pertaining to the Ar 3s binding energy region marked by two black bars, before (black symbols) and after (red symbols) background subtraction. Regions marked by red bars in (b) are used for background determination. Estimated background intensity is shown by a red trace in (c). See text for details. Intensity is expressed as coincident events/pixel of $30 \mathrm{meV}^{2}$ (b) or as coincident events per interval of $30 \mathrm{meV}$ (a),(c). In total, approx. $1.2 \times 10^{5}$ events are shown. The color scale of (b) is linear. 
Table 5: Properties of the Ar and ArKr cluster spectra (see also Tab. 1). Here, $N$ is $N_{\mathrm{Ar}}$ or $N_{\mathrm{ArKr}}$, as appropriate. $\alpha_{a u}$ denotes the ETMD3 decay efficiency, see text for details. n.d.: 'not determined'.

\begin{tabular}{lcccc} 
& $\mathrm{Kr}_{\text {in }}(\%)$ & $\langle N\rangle$ & $E_{b}(3 \mathrm{~s})(\mathrm{eV})$ & $\alpha_{a u}$ \\
\hline $\mathrm{Ar}$ & - & 7000 & $28.64(3)$ & - \\
$\mathrm{ArKr}$ & 3.0 & 109 & $28.77(3)$ & n.d. \\
$\mathrm{ArKr}$ & 5.0 & 6100 & $28.55(4)$ & $0.96(8)$ \\
$\mathrm{ArKr}$ & 5.0 & 1800 & $28.63(4)$ & $0.91(8)$ \\
\hline
\end{tabular}

ing the faster and $e_{2}$ the slower electron. As the binding energies of Ar 3s states in Ar clusters and mixed ArKr clusters are known (Tab. 5), we can identify the part of the secondary electron spectrum which can be attributed to emission from an $\mathrm{Ar} 3 \mathrm{~s}^{-1}$ photoionized state (region marked by black bars in the Figure). It can clearly be discerned that this state decays by electron emission, with a secondary electron spectrum which is very low in energy, and peaks at the lowest kinetic energy of $e_{2}$ that could be measured (approx. $100 \mathrm{meV}$ ). No such autoionization feature is present in pure Ar clusters (Fig. 12). There, the double ionization threshold of the cluster is too high in energy to energetically allow an ICD or ETMD [74, 75].

Some background at other combinations of energies can be seen in Fig. 11 (b), and is attributed to inelastic electron scattering, or decay of excitonic satellites, which are underlying the Ar 3s threshold in larger Ar clusters $[76,77]$. (In principle also ICD of electron correlation satellites can appear in this region, in the Ar dimer however the lowest such satellite has a binding energy of $32.2 \mathrm{eV}$ (above the excitation energy used here) [75].) In order to 


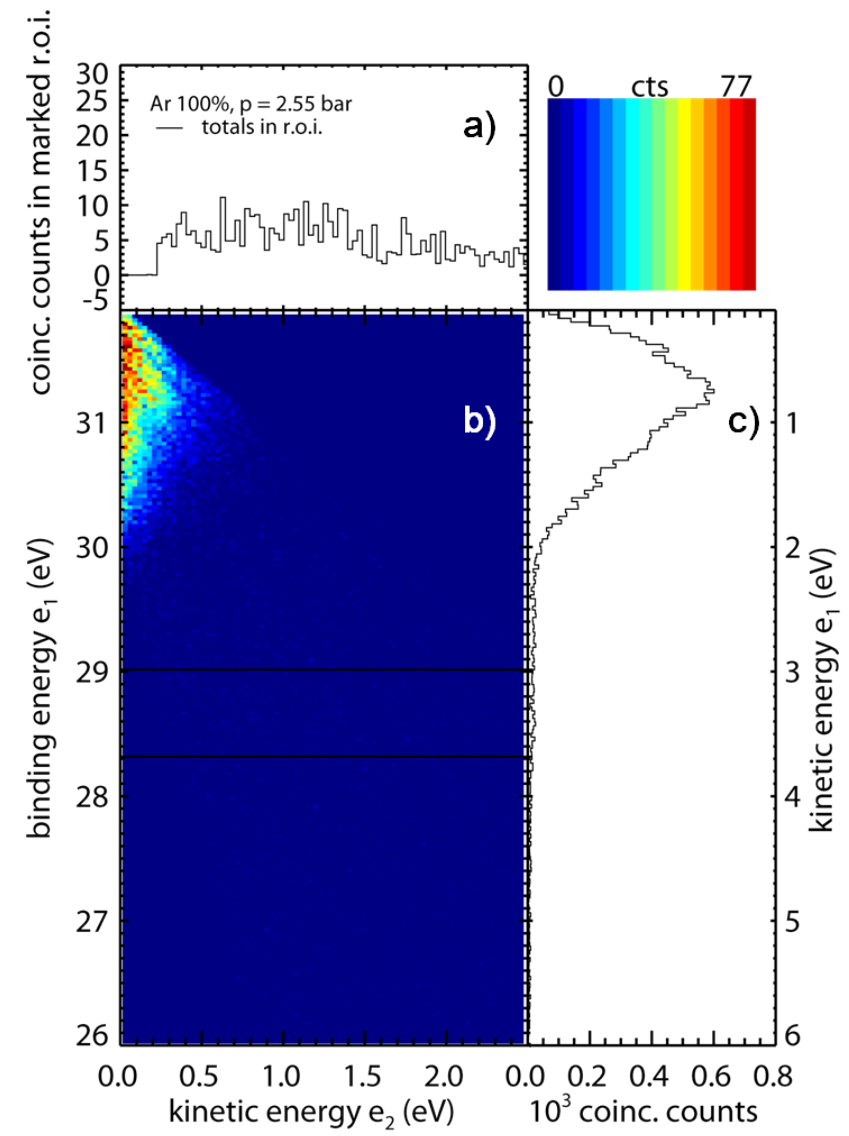

Figure 12: Photon excited electron-electron coincidence spectrum of pure Ar clusters in the region of Ar inner valence electrons. See Fig. 11 for labelling. 
disentangle the contribution of the background from our signal, we divided the coincident electron signals into narrow intervals of $e_{2}$ energy, and, for each interval, determined an appropriate background for the Ar 3s region by a polynomial fit to the signal in the two background regions marked by pairs of red bars in Fig. 11 (b). This background was subtracted to give the lower (red) data set shown in Fig. 11 (a). The sum of all background polynomials that were subtracted is shown as the solid, red trace in Fig. 11 (c). We will discuss the shape of the secondary electron spectrum in some more detail below, but note here the good agreement with our simulations (Fig. 9). This corroborates the assignment of this decay process to ETMD3, which was given in Ref. [37] based solely on energetical arguments.

In total, the signal from inelastic scattering (and, possibly, excitonic satellites) makes up a larger fraction of the two electron emission than ETMD3. A similar result has been quantitatively discussed for ICD in Ne clusters of different size [78].

In Figure 13, for comparison we again present the experimental ETMD3spectrum and the spectrum simulated for the mixed core-shell systems. For the experimental data, the error was calculated as the weighted sum of the statistical error of the estimated background and the statistical error of the spectrum before background subtraction. Given that our calculations still contain some simplifying assumptions in order to make the problem tractable, e.g. the representation of the initial and final single particle energies by simple numbers, we consider the amount of agreement very satisfactory. Since the total decay width could not be measured in this experiment, the comparison can only extend to the shape of the spectra. For example, experimentally 


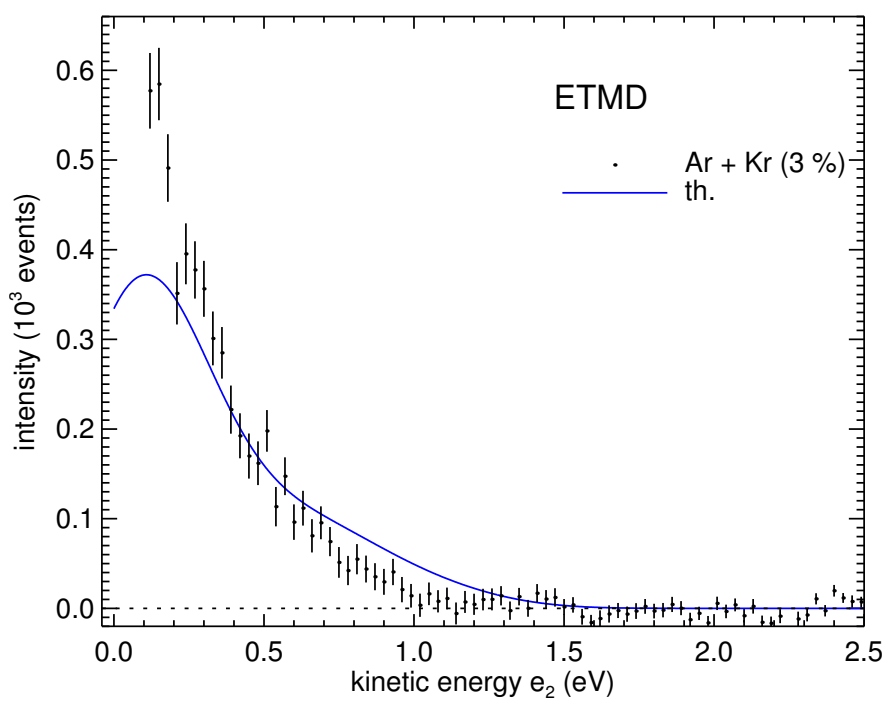

Figure 13: ETMD spectrum, after background subtraction, from measurements of mixed Ar-Kr clusters with $3 \%$ krypton in the initial gas mixture (corresponding to approximately $37 \%$ krypton in the final cluster). Data are the same as in panel (a) of Fig. 11. For comparison, we also show the data calculated for a mixed core-shell system from Fig. 9 (cluster structure in the right panel of Fig. 8), normalized to the total area of the experimental data. 
we cannot decide on the factor of four increase in decay probability between the strict core-shell system and the structure with sub-surface Ar.

From a comparison of our calculated ETMD3 lifetime to the one from fluorescence in pure Ar gas, we suggested that ETMD3 is the dominating decay channel. We checked this assertion experimentally with the methodology described in [56]. Briefly, for a feature in the $e_{1}$ spectrum, e.g. the Ar 3s primary photoelectron line, we perform the ratio of coincident to total counts. After correction with the detection efficiency this should give unity, if all primary excited states decay by emission of a second electron. We took the respective peak areas from least squares fits to the coincident and single hit inner valence spectra (not shown, see [41] for a similar study), and corrected the result by a detection efficiency of 0.27 , determined as outlined in [51]. The result of this exercise gives $\alpha_{a u}$, the fraction of $\mathrm{Ar}_{3 \mathrm{~s}^{-1}}$ ionized states which decay by emission of a low energy electron, and is shown in Tab. 5; the data set with $3 \% \mathrm{Kr}$ admixture could not be analyzed because its degree of condensation was too low. In other words, we have shown experimentally that the decay probability due to ETMD3 for the ArKr system is practically equal to one. Although the result for the smaller clusters seems to be somewhat lower, given the experimental error we would not like to further interpret this difference.

Finally, for comparison we briefly discuss the electron-electron coincidence spectrum of pure Kr clusters (Fig. 14). No autoionization features specific to the region of Ar 3s binding energies can be seen here. Instead, two-electron emission seems to be dominated by electron pairs with a very unequal energy sharing, and total kinetic energies in the range 3-5.5 eV (two-hole final state 


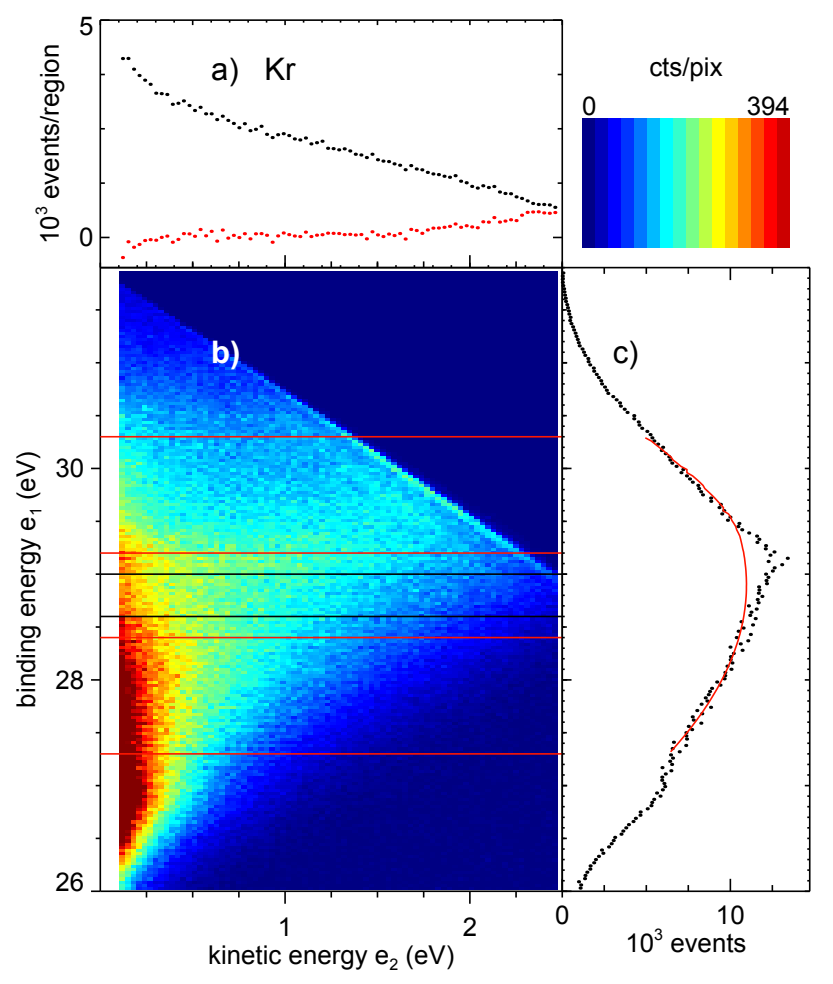

Figure 14: Photon excited electron-electron coincidence spectrum of pure Kr clusters in the region of Ar inner valence electrons. See Fig. 11 for labelling. 
energy 26.5-29 eV). Following the discussion elsewhere one could attribute this intensity to sequential emission of two electrons by inelastic electron scattering. The diagonal corresponding to the minimum total final state energy for this mechanism can be clearly seen in Fig. 14 (b). Nevertheless, this panel also shows a propensity for emission of electron pairs with a very low energy $(<0.4 \mathrm{eV}) e_{2}$ electron. As inelastic electron scattering rather leads to an equipartitioning of all energy combinations, we attribute this part of the two-electron intensity to autoionization of $\mathrm{Kr}$ satellite states. In rare gas dimers, ICD of the cluster states derived from the atomic correlation and shake-up satellite spectrum was indeed seen [75], but binding energies of these satellites are above the interval discussed here. (This type of satellite however could be responsible for the small feature seen at an $e_{1}$ binding energy of $29.3 \mathrm{eV}$ for both ArKr and pure Ar, see Fig.s 11 (c) and 14 (c).) Rather we attribute the discussed two-electron intensity to excitonic satellites having no atomic counterpart, which are clearly present in the relevant binding energy region (spectra not shown), in analogy to the discussion for Ar in [76]. It is interesting that here this autoionization driven channel seems well be able to compete with inelastic scattering, even at a cluster size of 700 . With lesser intensity, this process is also observed in larger ArKr clusters [37, 55].

\section{Summary}

We described the ETMD3 process after Ar 3s ionization in mixed ArKr clusters. We showed, that despite the necessity to describe this system including spin-orbit coupling, the overall ETMD electron spectrum and total decay width are very similar also in a non-relativistic description. In the rela- 
tivistic description the ArKr4p $\mathrm{p}_{3 / 2}^{-1} \mathrm{Kr}_{4} \mathrm{p}_{3 / 2}^{-1}$ channel is dominant. We quantified the geometry dependence of the channel opening, which was previously observed for the $\mathrm{ArKr}_{2}$ trimer. Systematic calculations of the decay width for different trimer angles clarified the role of orbital overlap and of the energy transfer and charge transfer distances. The results show that mixed cluster structures allowing for $\mathrm{ArKr}_{2}$ triples with short interatomic distances and large angles are beneficial for the $\operatorname{ETMD}(3)$ efficiency.

The calculated energies of the ETMD3 spectrum for larger clusters are in excellent agreement with the experimental results presented here and in Ref. [37]. We therefore conclude that the asymptotic approach is a useful tool to study the ETMD3 process in clusters, and might find applications also in liquids.

\section{Acknowledgements}

EF acknowledges funding from the Research Council of Norway through a Centre of Excellence Grant (Grant No. 179568/V30). We thank HZB for the allocation of synchrotron radiation beamtime, and the Deutsche Forschungsgemeinschaft for funding via the Forschergruppe 1789. MF and UH would like to thank Nicolas Sisourat for inspiring discussions while these experiments were done. 
[1] L. Meitner, Über die Entstehung der $\beta$-Strahl-Spektren radioaktiver Substanzen, Z. Phys. 9 (1922) 131.

[2] P. Auger, Sur les rayons $\beta$ secondaires produits dans un gaz par des rayons x, C. R. Acad. Sci. 177 (1923) 169.

[3] C. C. Chang, Auger electron spectroscopy, Surface Science 25 (1) (1971) 53.

[4] L. E. Davis (Ed.), Modern Surface Analysis: Metallurgical Applications of Auger Electron Spectroscopy (AES) and X-ray Photoelectron Spectroscopy (XPS)., The Metallurgical Society of AIME, 1980.

[5] C. Linsmeier, H. Knozinger, E. Taglauer, Ion-scattering and augerelectron spectroscopy analysis of alumina-supported rhodium model catalysts, Surface Science 275 (1-2) (1992) 101.

[6] L. S. Cederbaum, J. Zobeley, F. Tarantelli, Giant Intermolecular Decay and Fragmentation of Clusters, Phys. Rev. Lett. 79 (24) (1997) 4778.

[7] S. Marburger, O. Kugeler, U. Hergenhahn, T. Möller, Experimental evidence for interatomic coulombic decay in ne clusters, Phys. Rev. Lett. 90 (20) (2003) 203401.

[8] G. Öhrwall, M. Tchaplyguine, M. Lundwall, R. Feifel, H. Bergersen, T. Rander, A. Lindblad, J. Schulz, S. Peredkov, S. Barth, S. Marburger, U. Hergenhahn, S. Svensson, O. Björneholm, Femtosecond Interatomic Coulombic Decay in Free Neon Clusters: Large Lifetime Differences between Surface and Bulk, Phys. Rev. Lett. 93 (17) (2004) 173401. 
[9] T. Jahnke, A. Czasch, M. S. Schöffler, S. Schössler, A. Knapp, M. Käsz, J. Titze, C. Wimmer, K. Kreidi, R. E. Grisenti, A. Staudte, O. Jagutzki, U. Hergenhahn, H. Schmidt-Böcking, R. Dörner, Experimental observation of interatomic coulombic decay in neon dimers, Phys. Rev. Lett. 93 (16) (2004) 163401.

[10] U. Hergenhahn, Interatomic and Intermolecular Coulombic Decay: The Early Years, J. Electron Spectrosc. Relat. Phenom. 184 (2011) 78.

[11] T. Jahnke, Interatomic and intermolecular Coulombic decay: the coming of age story, J. Phys. B: Atomic, Molecular and Optical Physics 48 (2015) 082001.

[12] S. Barth, S. Joshi, S. Marburger, V. Ulrich, A. Lindblad, G. Öhrwall, O. Björneholm, U. Hergenhahn, Observation of resonant Interatomic Coulombic Decay in Ne clusters, J. Chem. Phys. 122 (24) (2005) 241102.

[13] T. Aoto, K. Ito, Y. Hikosaka, E. Shigemasa, F. Penent, P. Lablanquie, Properties of resonant interatomic coulombic decay in ne dimers, Phys. Rev. Lett. 97 (2006) 243401.

[14] K. Gokhberg, V. Averbukh, L. S. Cederbaum, Interatomic decay of inner-valence-excited states in clusters, J. Chem. Phys. 124 (2006) 144315.

[15] S. Kopelke, K. Gokhberg, L. S. Cederbaum, V. Averbukh, Calculation of resonant interatomic coulombic decay widths of inner-valence-excited states delocalized due to inversion symmetry, J. Chem. Phys. 130 (2009) 144103. 
[16] S. Kopelke, K. Gokhberg, V. Averbukh, A. Tarantelli, L. S. Cederbaum, $\mathrm{Ab}$ initio interatomic decay widths of excited states by applying stieltjes imaging to lanczos pseudospectra, J. Chem. Phys. 134 (2011) 094107.

[17] Y. Morishita, X.-J. Liu, N. Saito, T. Lischke, M. Kato, G. Prümper, M. Oura, H. Yamaoka, Y. Tamenori, I. H. Suzuki, K. Ueda, Experimental Evidence of Interatomic Coulombic Decay from the Auger Final States in Argon Dimers, Phys. Rev. Lett. 96 (24) (2006) 243402.

[18] S. D. Stoychev, A. I. Kuleff, F. Tarantelli, L. S. Cederbaum, On the interatomic electronic processes following Auger decay in neon dimer, J. Chem. Phys. 129 (7) (2008) 074307.

[19] P. Kolorenc, V. Averbukh, K. Gokhberg, L. S. Cederbaum, Ab initio calculation of interatomic decay rates of excited doubly ionized states in clusters, J. Chem. Phys. 129 (2008) 244102.

[20] K. Kreidi, T. Jahnke, T. Weber, T. Havermeier, X. Liu, Y. Morisita, S. Schössler, L. Schmidt, M. Schöffler, M. Odenweller, N. Neumann, L. Foucar, J. Titze, B. Ulrich, F. Sturm, C. Stuck, R. Wallauer, S. Voss, I. Lauter, H. K. Kim, M. Rudloff, H. Fukuzawa, G. Prümper, N. Saito, K. Ueda, A. Czasch, O. Jagutzki, H. Schmidt-Böcking, S. S., P. V. Demekhin, R. Dörner, Relaxation processes following 1s photoionization and Auger decay in Ne2, Phys. Rev. A 78 (4) (2008) 043422.

[21] T. Miteva, Y.-C. Chiang, P. Kolorenč, A. I. Kuleff, K. Gokhberg, C. L. S., Interatomic Coulombic decay following resonant core excitation of Ar in argon dimer, J. Chem. Phys. 141 (2014) 064307. 
[22] A. Bande, Electron dynamics of interatomic coulombic decay in quantum dots induced by a laser field, J. Chem. Phys. 138 (2013) 214104.

[23] P. H. P. Harbach, M. Schneider, S. Faraji, A. Dreuw, Intermolecular coulombic decay in biology: the initial electron detachment from fadhin dna photolyases, J. Phys. Chem. Lett. 4 (2013) 943.

[24] E. Alizadeh, T. M. Orlando, L. Sanche, Biomolecular Damage Induced by Ionizing Radiation: The Direct and Indirect Effects of Low-Energy Electrons on DNA, Annu. Rev. Phys. Chem. 66 (2015) 379.

[25] K. Gokhberg, P. Kolorenč, A. I. Kuleff, L. S. Cederbaum, Siteand energy-selective slow-electron production through intermolecular Coulombic decay, Nature 505 (2014) 661.

[26] F. Trinter, M. S. Schöffler, H. K. Kim, F. P. Sturm, K. Cole, N. Neumann, A. Vredenborg, J. Williams, I. Bocharova, R. Guillemin, M. Simon, A. Belkacem, A. L. Landers, T. Weber, H. Schmidt-Böcking, R. Dörner, J. T., Resonant Auger decay driving intermolecular Coulombic decay in molecular dimers, Nature 505 (2014) 664.

[27] R. Santra, J. Zobeley, L. S. Cederbaum, Electronic decay of valence holes in clusters and condensed matter, Phys. Rev. B 64 (24) (2001) 245104.

[28] E. Fasshauer, M. Pernpointner, K. Gokhberg, Interatomic decay of inner-valence ionized states in arxe clusters: Relativistic approach, J. Chem. Phys. 138 (2013) 014305. 
[29] E. Fasshauer, M. Förstel, S. Pallmann, M. Pernpointner, U. Hergenhahn, Using icd for structure analysis of clusters: a case study on coexpanded ne-ar clusters, New J. Phys. 16 (2014) 103026.

[30] K. t. GokhbergTo be published.

[31] E. Fasshauer, Non-nearest neighbour icd in clusters, New J. Phys. 18 (2016) 043028.

[32] C. Buth, R. Santra, L. S. Cederbaum, Impact of interatomic electronic decay processes on Xe $4 \mathrm{~d}$ hole decay in the xenon fluorides, J. Chem. Phys. 119 (2003) 10575-10584.

[33] J. Zobeley, R. Santra, L. S. Cederbaum, Electronic decay in weakly bound heteroclusters: Energy transfer versus electron transfer, J. Chem. Phys. 115 (11) (2001) 5076.

[34] R. Santra, L. S. Cederbaum, Non-Hermitian electronic theory and applications to clusters, Phys. Reports 368 (1) (2002) 1.

[35] V. Stumpf, N. V. Kryzhevoi, K. Gokhberg, L. S. Cederbaum, Enhanced one-photon double ionization of atoms and molecules in an environment of different species, Phys. Rev. Lett. 112 (2014) 193001.

[36] V. Stumpf, K. Gokhberg, L. S. Cederbaum, The role of metal ions in X-ray-induced photochemistry, Nature Chemistry 8 (2016) 237.

[37] M. Förstel, M. Mucke, T. Arion, A. M. Bradshaw, U. Hergenhahn, Autoionization mediated by electron transfer, Phys. Rev. Lett. 106 (2011) 033402. 
[38] I. Unger, R. Seidel, S. Thürmer, E. F. Aziz, L. S. Cederbaum, E. Muchová, P. Slavíček, B. Winter, N. V. Kryzhevoi, First Observation of Electron Transfer Mediated Decay in Aqueous Solutions: A Novel Probe of Ion Pairing, Nat. Chem. in print.

[39] K. Sakai, S. Stoychev, T. Ouchi, I. Higuchi, M. Schöffler, T. Mazza, H. Fukuzawa, K. Nagaya, M. Yao, Y. Tamenori, A. I. Kuleff, N. Saito, K. Ueda, Electron-transfer-mediated decay and interatomic coulombic decay from the triply ionized states in argon dimers, Phys. Rev. Lett. 106 (2011) 033401. doi:http://dx.doi.org/10.1103/PhysRevLett.106.033401.

[40] M. Pernpointner, N. V. Kryzhevoi, S. Urbaczek, Possible electronic decay channels in the ionization spectra of small clusters composed of $\mathrm{Ar}$ and Kr: A four-component relativistic treatment, J. Chem. Phys. 129 (2008) 024304.

[41] M. Förstel, M. Mucke, T. Arion, T. Lischke, M. Pernpointner, U. Hergenhahn, E. Fasshauer, to be published.

[42] Y. Morishita, N. Saito, I. H. Suzuki, H. Fukuzawa, X.-J. Liu, K. Sakai, G. Prümper, K. Ueda, H. Iwayama, K. Nagaya, M. Yao, K. Kreidi, M. Schöffler, T. Jahnke, S. Schössler, R. Dörner, T. Weber, J. Harries, Y. Tamenori, Evidence of interatomic Coulombic decay in ArKr after Ar 2p Auger decay, J. Phys. B: Atomic, Molecular and Optical Physics 41 (2008) 025101.

[43] K. Ueda, H. Fukuzawa, X.-J. Liu, K. Sakai, G. Prümper, Y. Morishita, N. Saito, I. H. Suzuki, K. Nagaya, H. Iwayama, M. Yao, K. Kreidi, 
M. Schöffler, T. Jahnke, S. Schössler, R. Dörner, T. Weber, J. Harries, Y. Tamenori, Interatomic Coulombic decay following the Auger decay: Experimental evidence in rare-gas dimers, J. Electron Spectrosc. Relat. Phenom. 166-167 (2008) 3.

[44] K. Kimura, H. Fukuzawa, T. Tachibana, Y. Ito, S. Mondal, M. Okunishi, M. Schöffler, J. Williams, Y. Jiang, Y. Tamenori, N. Saito, K. Ueda, Controlling Low-energy Electron Emission via Resonant-Auger-induced Interatomic Coulombic Decay, J. Phys. Chem. Lett. 4 (2013) 1838.

[45] T. Miteva, Y.-C. Chiang, P. Kolorenč, A. I. Kuleff, C. L. S., K. Gokhberg, The effect of the partner atom on the spectra of interatomic Coulombic decay triggered by resonant Auger processes, J. Chem. Phys. 141 (2014) 164303.

[46] G. Wentzel, Über strahlungslose quantensprünge, Z. Physik 43 (1927) 524.

[47] H. Feshbach, Unified theory of nuclear reactions, Ann. Phys. 5 (1958) 357.

[48] H. Feshbach, A unified theory of nuclear reactions. ii, Ann. Phys. 19 (1962) 287.

[49] U. Fano, Effects of configuration interaction on intensities and phase shifts, Phys. Rev. 124 (6) (1961) 1866-1878.

[50] T. Arion, M. Mucke, M. Förstel, A. M. Bradshaw, U. Hergenhahn, Interatomic Coulombic decay in mixed NeKr clusters, J. Chem. Phys. 134 (2011) 074306. 
[51] M. Mucke, M. Förstel, T. Lischke, T. Arion, A. M. Bradshaw, U. Hergenhahn, Performance of a short "magnetic bottle" electron spectrometer., Rev. Sci. Instrum. 83 (6) (2012) 063106. doi:http://dx.doi.org/10.1063/1.4729256.

[52] O. F. Hagena, Cluster ion sources, Rev. Sci. Instrum. 63 (1992) 23742379 .

[53] O. G. Danylchenko, S. I. Kovalenko, O. P. Konotop, V. N. Samovarov, Diagnostics of composition and size of clusters formed in supersonic jets of Ar-Kr gas mixtures, Low Temp. Phys. 41 (8) (2015) 637-644. doi:10.1063/1.4928921.

[54] G. V. Marr, J. B. West, Absolute photoionization cross-section tables for helium, neon, argon and krypton in the vuv spectral regions, At. Data Nucl. Data Tables 18 (1976) 497.

[55] M. Förstel, Investigation of non-local autoionization processes in rare gas clusters, Ph.D. thesis, TU Berlin (2012).

URL http://opus.kobv.de/tuberlin/volltexte/2012/3656/

[56] M. Förstel, T. Arion, U. Hergenhahn, Measuring the efficiency of interatomic Coulombic decay in Ne clusters, J. Electron Spectrosc. Relat. Phenom. $191 \quad$ (2013) 16. doi:http://dx.doi.org/10.1016/j.elspec.2013.11.002.

[57] HARDRoC, Hunting Asymptotic Relativistic Decay Rates of Clusters (2013), written by E. Fasshauer (see http://www.pci.uniheidelberg.de/tc/usr/elke/hardroc/html/main.html). 
[58] J. Thyssen, T. Fleig, H. J. A. Jensen, A direct relativistic fourcomponent multiconfiguration self-consistent-field method for molecules, J. Chem. Phys. 129 (2008) 034109.

[59] T. Fleig, J. Olsen, L. Visscher, The generalized active space concept for the relativistic treatment of electron correlation. ii. large-scale configuration interaction implementation based on relativistic 2- and 4-spinors and its application, J. Chem. Phys. 119 (2003) 2963.

[60] S. R. Knecht, Parallel relativistic multiconfiguration methods: New powerful tools for heavy-element electronic-structure studies, Ph.D. thesis, University of Duesseldorf (2009).

[61] DIRAC, a relativistic ab initio electronic structure program, Release DIRAC15 (2015), written by R. Bast, T. Saue, L. Visscher, and H. J. Aa. Jensen, with contributions from V. Bakken, K. G. Dyall, S. Dubillard, U. Ekstroem, E. Eliav, T. Enevoldsen, E. Fasshauer, T. Fleig, O. Fossgaard, A. S. P. Gomes, T. Helgaker, J. Henriksson, M. Ilias, Ch. R. Jacob, S. Knecht, S. Komorovsky, O. Kullie, J. K. Laerdahl, C. V. Larsen, Y. S. Lee, H. S. Nataraj, M. K. Nayak, P. Norman, G. Olejniczak, J. Olsen, Y. C. Park, J. K. Pedersen, M. Pernpointner, R. Di Remigio, K. Ruud, P. Salek, B. Schimmelpfennig, J. Sikkema, A. J. Thorvaldsen, J. Thyssen, J. van Stralen, S. Villaume, O. Visser, T. Winther, and S. Yamamoto (see http://www.diracprogram.org).

[62] J. A. R. Samson, J. L. Gardner, A. F. Starace, ${ }^{2} P_{3 / 2}:{ }^{2} P_{1 / 2}$ partial photoionization cross-section ratios in the rare gases, Phys. Rev. A 12 (1975) 1459. 
[63] A. Bondi, van der waals volumes and radii, J. Phys. Chem. 68 (1964) 441.

[64] C. Kittel, Introduction to Solid State Physics, eigth Edition, University of California, Berkeley, 2005.

[65] M. Lundwall, W. Pokapanich, H. Bergersen, A. Lindblad, T. Rander, G. Öhrwall, M. Tchaplyguine, S. Barth, U. Hergenhahn, S. Svensson, O. Björneholm, Self-assembled heterogeneous argon/neon core-shell clusters studied by photoelectron spectroscopy, J. Chem. Phys. 126 (2007) 214706.

[66] M. Tchaplyguine, M. Lundwall, M. Gisselbrecht, G. Öhrwall, R. Feifel, S. Sorensen, S. Svensson, N. Mårtensson, O. Björneholm, Phys. Rev. A 69 (2004) 031201.

[67] M. Lundwall, M. Tchaplyguine, G. Öhrwall, R. Feifel, A. Lindblad, A. Lindgren, S. Sorensen, S. Svensson, O. Björneholm, Chem. Phys. Lett. 392 (2004) 433.

[68] H. Vach, Solvation effects in large binary van der Waals clusters with high doping rates, J. Chem. Phys. 113 (3) (2000) 1097-1103.

[69] M. Lundwall, H. Bergersen, A. Lindblad, G. Öhrwall, M. Tchaplyguine, S. Svensson, O. Björneholm, Phys. Rev. A 74 (2006) 043206.

[70] Avogadro: an open-source molecular builder and visualization tool. Version 1.1.0. http://avogadro.openmolecules.net. 
[71] M. D. Hanwell, D. E. Curtis, D. C. Lonie, T. Vandermeersch, E. Zurek, G. R. Hutchison, Avogadro: An advanced semantic chemical editor, visualization, and analysis platform, J. Cheminformatics 4 (2012) 17.

[72] S. Lauer, H. Liebel, F. Vollweiler, H. Schmoranzer, B. M. Lagutin, P. V. Demekhin, I. D. Petrov, V. L. Soukhorukov, Lifetimes of the $n \mathrm{~s}^{1} n \mathrm{p}^{6}$ ${ }^{2} \mathrm{~S}_{\text {frac12 }}$ states of singly ionized argon, krypton and xenon, J. Phys. B: At. Mol. Opt. Phys. 32 (1999) 2015.

[73] M. Mucke, M. Braune, S. Barth, M. Förstel, T. Lischke, V. Ulrich, T. Arion, U. Becker, A. Bradshaw, U. Hergenhahn, A hitherto unrecognized source of low-energy electrons in water, Nature Physics 6 (2010) 143.

[74] E. Rühl, C. Schmale, H. C. Schmelz, H. Baumgärtel, The double ionization potentials of argon clusters, Chem. Phys. Lett. 191 (5) (1992) 430-434.

[75] P. Lablanquie, T. Aoto, Y. Hikosaka, Y. Morioka, F. Penent, K. Ito, Appearance of interatomic Coulombic decay in Ar, Kr, and Xe homonuclear dimers, J. Chem. Phys. 127 (15) (2007) 154323.

[76] U. Hergenhahn, A. Kolmakov, M. Riedler, A. R. B. de Castro, O. Löfken, T. Möller, Observation of excitonic satellites in the photoelectron spectra of Ne and Ar clusters, Chem. Phys. Lett. 351 (2002) 235-241.

[77] A. Hans, A. Knie, M. Förstel, P. Schmidt, P. Reiß, C. Ozga, U. Hergenhahn, A. Ehresmann, Determination of absolute cross sections for cluster-specific decays, J. Phys. B At. Mol. Opt. Phys. 49 (10) (2016) 105101. doi:10.1088/0953-4075/49/10/105101. 
${ }_{793}$ [78] M. Mucke, T. Arion, M. Förstel, T. Lischke, U. Hergenhahn, Competition of inelastic electron scattering and Interatomic Coulombic Decay

795 in Ne clusters, J. Electron Spectrosc. Relat. Phenom. 200 (2015) 232. 